\title{
Analytic Scattering Theory for Many-Body Systems Below the Smallest Three-Body Threshold
}

\author{
Erik Balslev \\ Matematisk Institut, Universitetsparken Ny Munkegade, DK-8000 Aarhus C, Denmark
}

\begin{abstract}
We consider the Schrödinger operator $H=H_{0}+V$ of a many-body system, where $V$ is a sum of dilation-analytic, short range (not necessarily local) two-body interactions, together with the associated self-adjoint analytic family $H(z),|\operatorname{Arg} z|<a$, of complex-dilated operators. For each $z$ we construct the local wave operators and the S-matrix below the smallest 3-body threshold, using abstract stationary scattering theory and the Weinberg-van Winter equation. The diagonal element of the inverse S-matrix describing scattering within the channel $\alpha$ in the lowest energy range is proved to be the boundary value of a meromorphic function $\mathscr{S}_{\alpha}(z),-a<\operatorname{Arg} z<0$, where $\mathscr{S}_{\alpha}(z)$ is the S-matrix for $H(z)$ on the corresponding cut. Generally, the poles of $\mathscr{S}_{\alpha}(z)$ are resolvent resonances, but a resolvent resonance may not be a pole of $\mathscr{S}_{\alpha}(z)$, if it is embedded as an eigenvalue in the continuum of $H\left(z_{0}\right)$ for a suitable $z_{0}$.
\end{abstract}

\section{Introduction}

Scattering theory for two- and three-body Schrödinger operators with dilationanalytic potentials was studied in $[7,8]$. In [8] the abstract stationary theory developed by Howland [15], Kato [20], and Yajima [35] was utilized. As in these works the basic decomposition equation for the resolvent of the three-body problem was a type of symmetrized Faddeev equation. For the extension to the $n$-body problem we adopt an equation based on the Weinberg-van Winter equation (Lemmas 2.12 and 2.14). This has the advantage of simplicity and the disadvantage of the possibility of spurious poles in the resolvent equation. The effect of these, however, can on the whole be eliminated. Utilizing this equation, we construct wave- and scattering operators for the Schrödinger operator $H$ below the lowest three-body threshold $\Sigma_{3}^{0}$ as well as for the complex-dilated operators $H(z)$ and prove asymptotic completeness (Theorems 5.6 and 5.7). This is based on the limiting absorption principle of Sect. 3 and involves for the dilated operators $H(z)$ the construction of a spectral measure (Lemma 5.3). The spectral trace 
formalism of Kuroda [22] is utilized for the definition of the inverse wave operators (Definition 5.4). This yields a simple expression for the S-matrix (Theorem 5.10).

Perhaps the main importance of the dilation-analytic theory lies in its contribution to the analysis of resonances. In the spectral theory [4], the so-called resolvent resonances occur as poles of the resolvents of $H(z)$ and of certain analytically continued matrix element of these resolvents.

In the two-body case these resolvent resonances coincide with the poles of the analytically continued S-matrix [7]. In the many-body case this is not necessarily true. A resolvent resonance may be a continuum-embedded eigenvalue of $H(z)$ for a suitable $z$, in which case it generally does not give rise to a pole of the analytically continued S-matrix. More precisely, for each threshold $\lambda$ below $\Sigma_{3}^{0}$ it is shown, that the diagonal element $\left[\mathscr{S}^{-1}\left(\lambda+\frac{\varrho^{2}}{2 m_{D}}\right)\right]_{\lambda, \lambda}$ of the inverse S-matrix is the boundary value of a function $\mathscr{S}_{\lambda}\left(\varrho e^{i \varphi}\right)=\mathscr{S}_{\lambda}\left(\varphi, \frac{\varrho^{2}}{2 m_{D}}\right)$, meromorphic in an angle $\left\{z=\varrho e^{i \varphi} \mid\right.$ $-a<\varphi<0, \varrho>0\}$ (Theorem 5.11). The operators $\mathscr{S}_{\lambda}(\varphi, \mu)$ are scattering matrices for the dilated operators corresponding to the cut $\lambda+e^{2 i \varphi} \mathrm{R}^{+}$. It is then shown, that if $\kappa=\lambda+\frac{z^{2}}{2 m_{D}}$ is not a pole (or a spurious pole) of the resolvent on the other side of the cut, then $\kappa$ is a resolvent resonance if and only if $z$ is a pole of $\mathscr{S}_{\lambda}(\zeta)$ [Theorem 5.17(1)].

If $\kappa$ is an embedded eigenvalue of $H(z)$ however - in which case it appears as a resolvent resonance on both sides of the cut - then $\mathscr{S}_{\lambda}(\zeta)$ has a pole at $z$ if and only if there are also resonance eigenfunctions which do not continue analytically as eigenfunctions of $H(z)$ [Theorem 5.17(2)]. These results follow from an analysis of the behaviour of resonance eigenfunctions in the limit when the spectrum of $H(z)$ passes through the resonance, characterizing "embedded" eigenfunctions by the vanishing of a closely related function on the energy shell (Lemmas 4.6 and 4.7) and the establishment of an isomorphism of $\mathscr{N}\left(\mathscr{S}_{\lambda}^{-1}(z)\right)$ with the space of "nonembedded" resonance eigenfunctions (Theorems 5.13 and 5.15).

For simplicity we have assumed that thresholds are non-degenerate. In Sect. 6 we sketch the extension to the more general case, as it occurs when there are symmetries in the system.

Analyticity properties of the S-matrix have been established for 3- and 4-body Schrödinger operators by Hagedorn [13], who obtained continuation also of offdiagonal elements with poles at most at resonances under the assumption that the potentials are exponentially decaying and dilation-analytic. Other results in this direction are due to Nuttall and Singh [25] for many-body systems below $\Sigma_{3}^{0}$, including also Coulomb potentials. Results on general $n$-body scattering theory for the operators $H(z)$ have been announced by van Winter [34] and Sigal [28].

Although the emphasis is on the analytic theory, we remark that without the analyticity assumption the method yields a proof of completeness below $\Sigma_{3}^{0}$ in the framework of the abstract stationary theory under the assumption A1. In this generality, however, the singular points are only known, by a lemma of Kuroda [21], to lie in a closed set of measure zero. Completeness below $\Sigma_{3}^{0}$ has been 
proved under various conditions by the time-dependent methods of Combes [9], simplified and generalized by Simon [30] and of Enss [12]. Contributions to the general $n$-body problem are due to Hepp [14], using Faddeev-Yakubovski equations, and Sigal [27], using Berezin's equations.

\section{Notations and Basic Assumptions}

We consider a system of $n$ particles, denoted by $1, \ldots, n$, in $m$-dimensional space $\mathrm{R}^{m}$. The mass, position and momentum of particle $i$ in the center-of-mass frame are denoted by $m_{i}, x_{i}$, and $k_{i}$. We denote the pairs $(i j)$ by $\alpha, \beta$ etc. For $\alpha=(i j)$ we set

$$
x_{\alpha}=x_{j}-x_{i}, \quad k_{\alpha}=\left(m_{i}+m_{j}\right)^{-1}\left(m_{j} k_{i}-m_{i} k_{j}\right) .
$$

Proper subsystems or clusters are denoted by $C$, and for $C$ containing at least 2 particles $m_{c}, x_{c}$, and $k_{c}$ denote the total mass of $C$ and the set of linearly independent position and momentum vectors of particles in $C$ relative to the center-of-mass of $C$.

A cluster decomposition is a partition $D_{k}$ of the system into $k$ disjoint cluster $C_{1}, \ldots, C_{k}$. For $D_{k}=\left\{C_{1}, \ldots, C_{k}\right\}$ we denote by $y_{D_{k}}$ and $p_{D_{k}}$ the set of $k-1$ linearly independent relative position and momentum vectors of the centers-of-mass of clusters $C_{i}$ with the center-of-mass of the total system removed. We set $D=D_{2}$ $=\left\{C_{1}, C_{2}\right\}$. We write $D_{i} \subset C_{j}$ if each cluster of $D_{i}$ is contained in a cluster of $D_{j}$.

The basic Hilbert space $\mathscr{H}$ is $L^{2}\left(\mathrm{R}^{m(n-1)}\right)$, where we can choose any set of $n-1$ basis vectors for the underlying space of positions or momenta relative to the center-of-mass. We choose to work mainly in momentum representation, switching occasionally to position space in connection with some of the proofs. The two representations are distinguished by subscripts like $(x, y)$ for position space and $(k, p)$ for momentum space.

We denote by $\mathscr{H}^{c}$ the Hilbert space associated with the internal coordinates of $C$. If $C$ contains only one particle, we take $\mathscr{H}^{C}$ to be a 1 -dimensional space. We set $\mathscr{H}^{D_{k}}=\prod_{i=1}^{k} \otimes \mathscr{H}^{C_{\imath}}$ for $D_{k}=\left\{C_{1}, \ldots, C_{k}\right\}$, while $\mathscr{H}_{r}^{D_{k}}=L^{2}\left(R^{m(k-1)}\right)$ is the Hilbert space of relative positions or momenta of the centers-of-mass of $C_{1}, \ldots, C_{k}$.

The free Hamiltonian $H_{0}$ can be written in various ways depending on the choice of coordinates. For later use we shall give various explicit expressions of $H_{0}$.

Let $\alpha_{i}=\left(n_{i}^{1}, n_{i}^{2}\right), i=1, \ldots, n-1$, be a sequence of pairs, $M_{i}=\alpha_{1} \cup \ldots \cup \alpha_{i}$, and assume $n_{i}^{2} \notin M_{i-1}$, such that $\left(\alpha_{1}, \ldots, \alpha_{n-1}\right)$ defines a connected graph. Let $k_{i-1, i}$ be the relative momentum of the center-of-mass of $M_{i-1}$ and $n_{i}$ if $n_{i}^{1} \in M_{i-1}$ and the same with $n_{i}$ replaced by the center-of-mass of $\alpha_{i}$ if $n_{i}^{1} \notin M_{i-1}$. Set

$$
\mathscr{H}_{M_{i-1}, \alpha_{i}}=L^{2}\left(\mathrm{R}_{k_{i-1, i}}^{m}\right) \text { if } n_{i}^{1} \in M_{i-1}
$$

and

$$
\mathscr{H}_{M_{i-1}, \alpha_{i}}=L^{2}\left(\mathrm{R}_{k_{i-1}, k_{i}}^{m}\right) \otimes L^{2}\left(\mathrm{R}_{k_{\alpha_{i}}}^{m}\right) \quad \text { if } \quad n_{i}^{1} \notin M_{i-1} .
$$


In momentum space the free Hamiltonian $H_{0}^{M_{i-1}, \alpha_{i}}$ of the center-of-mass of $M_{i-1}$ and $n_{i}^{2}$ if $n_{i}^{1} \in M_{i-1}, \alpha_{i}$ if $n_{i}^{1} \notin M_{i-1}$, is given by

$$
H_{0, r}^{M_{i-1}, \alpha_{i}}=\frac{k_{i-1, i}^{2}}{2 m_{M_{i-1}, \alpha_{i}}},
$$

where $m_{M_{i-1}, \alpha_{i}}$ is the reduced mass of $M_{i-1}$ and $n_{i}^{2}$ if $n_{i}^{1} \in M_{i-1}$ and of $M_{i-1}$ and $\alpha_{i}$ if $n_{i}^{1} \in M_{i-1}$.

The free Hamiltonian $H_{0}^{\alpha_{i}}$ of $\alpha_{i}$ is given by

$$
H_{0}^{\alpha_{i}}=\frac{k_{\alpha_{i}}^{2}}{2 m_{\alpha_{i}}}, \quad m_{\alpha_{i}}^{-1}=m_{n_{i}^{1}}^{-1}+m_{n_{i}^{2}}^{-1} .
$$

Setting

$$
H_{0}^{M_{i-1}, \alpha_{i}}=\left\{\begin{array}{lll}
H_{0, r}^{M_{i-1}, \alpha_{i}} & \text { if } & n_{i}^{1} \in M_{i-1} \\
H_{0, r}^{M_{i-1}, \alpha_{2}}+H_{0}^{\alpha_{i}} & \text { if } & n_{i}^{1} \notin M_{i-1},
\end{array}\right.
$$

we can write the operator $H$ in the form

$$
H_{0}=H_{0}^{\alpha_{1}}+\sum_{i=2}^{n-1} H_{0}^{M_{i-1}, \alpha_{i}}
$$

where the variables are separated.

We set

$$
\begin{aligned}
R_{0, r}^{M_{2-1}, \alpha_{i}}(\zeta) & =\left(H_{0, r}^{M_{i-1}, \alpha_{2}}-\zeta\right)^{-1} \\
R_{0}^{\alpha_{2}}(\zeta) & =\left(H_{0}^{\alpha_{i}}-\zeta\right)^{-1} \\
R_{0}^{M_{i-1}, \alpha_{i}}(\zeta) & =\left(H_{0}^{M_{2-1}, \alpha_{i}}-\zeta\right)^{-1} \\
R_{0}(\zeta) & =\left(H_{0}-\zeta\right)^{-1}
\end{aligned}
$$

and

$$
H_{0}^{M_{\imath}}=H_{0}^{\alpha_{l}}+\sum_{j=2}^{i} H_{0}^{M_{j-1}, \alpha_{J}} .
$$

For any non-trivial cluster $C$ we denote by $H_{0}^{C}$ the free Hamiltonian of $C$ in the center-of-mass system. If $C$ contains only one particle, we let $H_{0}^{c}=0$ on the 1-dimensional space $\mathscr{H}^{C}$. For any $D_{k}=\left\{C_{1}, \ldots, C_{k}\right\}$ we denote by $H_{0, r}^{D_{k}}$ the free Hamiltonian of the centers of mass of $C_{1}, \ldots, C_{k}$. Then we have

$$
H_{0}=\sum_{i=1}^{k} H_{0}^{C_{i}}+H_{0, r}^{D_{k}} .
$$

We make use of the weighted Sobolev spaces $H^{s, \delta}\left(R^{l}\right)$ with differentiability parameter $s$ and weight $\delta$, as defined in [7], Sect. 1. For a discussion of the spaces $H^{s, \delta}\left(R^{l}\right)$ we refer to [23]. We set

$$
\begin{gathered}
H^{s}\left(\mathrm{R}^{l}\right)=H^{s, 0}\left(\mathrm{R}^{l}\right), \quad H^{s, \delta}=H^{s, \delta}\left(\mathrm{R}^{m(n-1)}\right), \\
H^{s, \delta}\left(R_{k_{\alpha}}^{m}\right)=H_{k_{\alpha}}^{s, \delta} \quad H^{s, \delta}\left(R_{p_{D}}^{m}\right)=H_{p_{D}}^{s, \delta} \text { etc. }
\end{gathered}
$$


For any pair of Hilbert spaces $\mathscr{H}_{1}, \mathscr{H}_{2}$ we denote by $\mathscr{B}\left(\mathscr{H}_{1}, \mathscr{H}_{2}\right)$ and $\mathscr{C}\left(\mathscr{H}_{1}, \mathscr{H}_{2}\right)$ the spaces of bounded and compact operators from $\mathscr{H}_{1}$ into $\mathscr{H}_{2}$.

We denote by $S^{l-1}$ the unit sphere in $\mathrm{R}^{l}$ and identify $L^{2}\left(\mathrm{R}^{l}\right)$ with $L^{2}\left(\mathrm{R}^{+}, L^{2}\left(S^{l-1}\right) ; \varrho^{l-1}\right)$, writing $f(\varrho, \cdot)$ for $f \in L^{2}\left(\mathrm{R}^{l}\right)$. The trace operators $\gamma(\varrho) \in \mathscr{B}\left(H^{s}\left(\mathrm{R}^{l}\right), L^{2}\left(S^{l-1}\right)\right)$ (see [23]) are defined for $s>1 / 2, \varrho>0$, by

$$
\gamma(\varrho) f=f(\varrho, \cdot) .
$$

For any $D=D_{2}$ we denote by $\gamma_{D}(\varrho)$ the trace operator in $\mathscr{B}\left(H^{s}\left(\mathrm{R}_{p_{D}}^{m}\right), L^{2}\left(S^{m-1}\right)\right)$. The unitary group of dilation operators $U(\varrho)$ is defined for $f \in \mathscr{H}, \varrho \in \mathrm{R}^{+}$, by

$$
(U(\varrho) f)(p)=\varrho^{\frac{m(n-1)}{2}} f(\varrho p) .
$$

For any operator $A$ in $\mathscr{H}$ we set $A(\varrho)=U(\varrho) A U\left(\varrho^{-1}\right)$. We denote by $U_{D}$ the dilation group on $\mathscr{H}_{r}^{D}$.

The dilation and trace operators are connected by

$$
\begin{aligned}
& \gamma(\varrho)=\varrho^{-\frac{m(n-1)}{2}} \gamma(1) U(\varrho), \\
& \gamma_{D}(\varrho)=\varrho^{-\frac{m}{2}} \gamma_{D}(1) U_{D}(\varrho) .
\end{aligned}
$$

For $0<a<\frac{\pi}{2}$, let $\mathcal{O}=\mathcal{O}_{a}$ denote the angular region

$$
\mathcal{O}_{a}=\left\{z=\varrho e^{i \varphi} \mid \varrho \in \mathrm{R}^{+},-a<\varphi<a\right\} .
$$

We use the notation

$$
(\langle\phi| f)\left(p_{D}\right)=\int_{\substack{\mathrm{R}_{k_{D}}^{m(n-2)} \\ m(n)}} f\left(k_{D}, p_{D}\right) \phi\left(k_{D}\right) d k_{D}
$$

whenever the right hand side is defined, and

$$
(|\phi\rangle g)\left(k_{D}, p_{D}\right)=\phi\left(k_{D}\right) g\left(p_{D}\right) .
$$

Assumptions on the Interaction

We make the following explicit assumption on the interaction $V=\sum_{\alpha} V_{\alpha}$.

A1. There exists $s>1 / 2$ such that

$$
V_{\alpha}=A_{\alpha} W_{\alpha} A_{\alpha} \text {, where } A_{\alpha}=\left(1-\Delta_{k \alpha}\right)^{-s / 2}
$$

and $W_{\alpha}$ is a symmetric operator in $L_{k_{\alpha}}^{2}$ such that

$$
W_{\alpha} \in \mathscr{C}\left(H_{k_{\alpha}}^{0,2}, L_{k_{\alpha}}^{2}\right) \text {. }
$$
to $\mathcal{O}$.

A2. The $\mathscr{C}\left(H_{k_{\alpha}}^{0,2}, L_{k_{\alpha}}^{2}\right)$-valued function $W_{\alpha}(\varrho)$ has an analytic extension from $\mathrm{R}^{+}$

A1 implies that $W_{\alpha}^{*} \in \mathscr{C}\left(L_{k_{\alpha}}^{2}, H_{k_{\alpha}}^{0,-2}\right)$, and by A2 $W_{\alpha}^{*}(\varrho)$ has an analytic extension to $\mathcal{O}$. Since $W_{\alpha} \leqq W_{\alpha}^{*}$, we shall simplify notation by writing

$$
W_{\alpha}(z) \in \mathscr{C}\left(H_{k_{\alpha}}^{0,2}, L_{k_{\alpha}}^{2}\right) \cap \mathscr{C}\left(L_{k_{\alpha}}^{2}, H_{k_{\alpha}}^{0,-2}\right) .
$$

The condition A1 was introduced by Kuroda [23]. 
For a discussion of the condition A2 for multiplicative potentials we refer to [3].

The operator $H_{0}$ is self-adjoint on $\mathscr{D}\left(H_{0}\right)=H^{0,2}$. It follows from A1 that $V_{\alpha}$ and hence $V$ is $H_{0}-\varepsilon$-bounded, so $H$ is self-adjoint on $\mathscr{D}\left(H_{0}\right)$. Moreover, by A2 the operators $H(z)=H_{0}(z)+V(z)$ form a self-adjoint, analytic family defined for $z \in \mathcal{O}$. We set

$$
\begin{aligned}
V^{C}(z) & =\sum_{\alpha \subset C} V_{\alpha}(z), \\
V_{D_{k}}(z) & =\sum_{i=1}^{k} \sum_{\alpha \subset C_{i}} V_{\alpha}(z), \\
V_{D_{k}}^{\prime}(z) & =V(z)-V_{D_{k}}(z), \\
H^{C}(z) & =H_{0}^{C}(z)+V^{C}(z), \quad H_{D_{k}}(z)=H_{0}(z)+\quad V_{D_{k}}(z), \\
R(z, \zeta) & =(H(z)-\zeta)^{-1} R^{C}(z, \zeta)=\left(H^{C}(z)-\zeta\right)^{-1} \\
R_{D_{k}}(z, \zeta) & =\left(H_{D_{k}}(z)-\zeta\right)^{-1} .
\end{aligned}
$$

We denote the set of all two-cluster decompositions $D$ by $\mathscr{D}$. For $D=\left\{C_{1}, C_{2}\right\} \in \mathscr{D}$ we set

$$
\sigma_{d}^{D}=\sigma_{d}\left(H^{C_{1}}+H^{C_{2}}\right)=\sigma_{d}\left(H^{C_{1}}\right)+\sigma_{d}\left(H^{C_{2}}\right),
$$

where $H^{C_{1}}+H^{C_{2}}=H^{C_{1}} \otimes I^{C_{2}}+I^{C_{1}} \otimes H^{C_{2}}$ acts in $\mathscr{H}^{C_{1}} \otimes \mathscr{H}^{C_{2}}$ :

Note that if $C_{2}$ is trivial, then $\sigma_{d}^{D}=\sigma_{d}\left(H^{C_{1}}\right)$.

We now make a further, simplifying assumption.

A3. For all $D \in \mathscr{D}$, such that $\sigma_{d}^{D} \neq \emptyset, \sigma_{d}^{D}$ consists of simple eigenvalues, and for $D_{1} \neq D_{2}, \sigma_{d}^{D_{1}} \cap \sigma_{d}^{D_{2}}=\emptyset$.

This assumption is made to simplify the treatment and presentation. In the last section the more general case, where A3 is not assumed, will be discussed.

The set of $k$-body thresholds $\Sigma_{k}(\varphi)$ is defined by

$$
\Sigma_{k}(\varphi)=\bigcup_{D_{k}}\left\{\sigma_{d}\left(H^{C_{1}}\left(e^{i \varphi}\right)\right)+\ldots+\sigma_{d}\left(H^{C_{k}}\left(e^{i \varphi}\right)\right)\right\}
$$

$\Sigma_{k}=\Sigma_{k}(0)$, in particular $\Sigma_{2}=\bigcup_{D} \sigma_{d}^{D}$.

We set

$$
\begin{aligned}
\Sigma_{k}^{0} & =\min \left\{\kappa \in \Sigma_{k}\right\}, \\
\Sigma(\varphi) & =\bigcup_{2 \leqq k \leqq n-1} \sigma_{k}(\varphi), \quad \Sigma=\Sigma(0) .
\end{aligned}
$$

For any $C$, we let $\Sigma^{C}(\varphi)$ denote $\Sigma(\varphi)$ for the operator $H^{C}\left(e^{i \varphi}\right)$.

Throughout this paper we fix $E$ as follows,

$$
E \in\left\{\kappa \in \Sigma_{2} \mid \kappa<\Sigma_{3}^{0}\right\}
$$

In case $\left\{\kappa \in \Sigma_{2} \mid \kappa<\Sigma_{3}^{0}\right\}$ is finite, $E=\Sigma_{3}^{0}$ is also allowed. Let

$$
\begin{aligned}
& \mathscr{D}_{E}=\left\{E \in \mathscr{D} \mid \sigma_{d}^{D} \cap\left[\Sigma_{2}^{0}, E\right) \neq \emptyset\right\} \\
& \mathscr{D}_{E}^{\prime}=\left\{D \in \mathscr{D} \mid \sigma_{d}^{D} \cap\left[\Sigma_{2}^{0}, E\right)=\emptyset\right\} \cup\left\{D_{k} \mid k \geqq 3\right\} .
\end{aligned}
$$


For $D \in \mathscr{D}_{E}$ let $n(D)$ be the number of points in $\sigma_{d}^{D} \cap\left[\Sigma_{2}^{0}, E\right)$, and let

$$
\lambda_{D}^{1}<\ldots<\lambda_{D}^{n(D)}, \quad \phi_{D}^{1}(z), \ldots, \phi_{D}^{n(D)}(z)
$$

be these eigenvalues with the corresponding normalized eigenfunctions chosen such that $\phi_{D}^{i}(z)$ is analytic in $\mathcal{O}$ [cf. Lemma 2.10(2)]. Let

$$
P_{D}^{i}(z)=\left|\phi_{D}^{i}(z)\right\rangle\left\langle\phi_{D}^{i}(\bar{z})\right|, \quad P_{D}^{E}(z)=\sum_{i=1}^{n(D)} P_{D}^{i}(z),
$$

and

$$
r_{D}^{i}(z, \zeta)=\left(\frac{z^{2} p_{D}^{2}}{2 m_{D}}+\lambda_{D}^{i}-\zeta\right)^{-1}, \quad i=1, \ldots, n(D)
$$

where $m_{D}^{-1}=m_{C_{1}}^{-1}+m_{C_{2}}^{-1}$, and $p_{D}$ is the relative momentum of the centers of mass of $C_{1}$ and $C_{2}$.

For $D \in \mathscr{D}_{E}$ we decompose $R_{D}(z, \zeta)$ with respect to $E$ by setting

$$
R_{D}(z, \zeta)=R_{D}^{E}(z, \zeta)+\sum_{i=1}^{n(D)}\left|\phi_{D}^{i}(z)\right\rangle r_{D}^{i}(z, \zeta)\left\langle\phi_{D}^{i}(\bar{z})\right|,
$$

where

$$
R_{D}^{E}(z, \zeta)=R_{D}(z, \zeta)\left(1-P_{D}^{H}(z)\right)
$$

We define the following Hilbert spaces and operators associated with the set of channels related to scattering below $E$.

To simplify notation, we set

$$
\begin{gathered}
\sum_{D \in \mathscr{D}_{E}} \sum_{i=1}^{n(D)}=\sum_{D, i} \\
\tilde{H}=\mathscr{H} \oplus \sum_{D, i} \oplus L_{p_{D}}^{2}
\end{gathered}
$$

with elements $\tilde{u}=\left(u,\left\{\tau_{D}^{i}\right\}\right)$

$$
\begin{aligned}
H^{s} & =H^{s} \oplus \sum_{D, i} \oplus H_{p_{D}}^{s}, \\
R_{1}(z, \zeta) & =R_{0}(z, \zeta) \oplus \sum_{D, i} \oplus r_{D}^{i}(z, \zeta), \\
J(z) \tilde{u} & =u+\sum_{D, i}\left|\phi_{D}^{i}(z)\right\rangle \tau_{D}^{i}, \quad \tilde{u} \in \tilde{\mathscr{H}}, \\
W_{D}^{i}(z) & =V_{D}^{\prime}(z)\left|\phi_{D}^{i}(z)\right\rangle, \\
W(z) \tilde{u} & =V(z) u+\sum_{D, i} W_{D}^{i}(z) \tau_{D}^{i}, \\
G_{D}^{i}(z, \zeta) & =\left|\phi_{D}^{i}(z)\right\rangle+W_{D}^{i}(z) r_{D}^{i}(z, \zeta), \\
G_{0}(z, \zeta) & =1+V(z) R_{0}(z, \zeta), \\
G(z, \zeta) \tilde{u} & =G_{0}(z, \zeta) u+\sum_{D, i} G_{D}^{i}(z, \zeta) \tau_{D}^{i}=\left[J(z)+W(z) R_{1}(z, \zeta)\right] \tilde{u} .
\end{aligned}
$$


For $\varphi \neq 0$ we also utilize another decomposition of $R_{D}(z, \zeta)$ corresponding to a single channel, associated with the threshold $\lambda=\lambda_{D}^{i}$. We write

$$
R_{D}(z, \zeta)=R_{D}^{\lambda}(z, \zeta)+\left|\phi_{D}^{i}(z)\right\rangle r_{D}^{i}(z, \zeta)\left\langle\phi_{D}^{i}(\bar{z})\right|
$$

where

$$
R_{D}^{\lambda}(z, \zeta)=R_{D}(z, \zeta)\left(1-P_{D}^{i}(z)\right)
$$

The relevant spaces and operators are redefined as $\tilde{\mathscr{H}}_{\lambda}=\mathscr{H} \oplus L_{p_{D}}^{2}$, $\tilde{H}_{\lambda}^{s}=H^{s} \oplus H_{p_{D}}^{s}, \tilde{u}=(u, \tau) \in \tilde{\mathscr{H}}_{\lambda}$

$$
\begin{aligned}
J_{\lambda}(z) \tilde{u} & =u+\left|\phi_{D}^{i}(z)\right\rangle \tau, \\
R_{1, \lambda}(z, \zeta) \tilde{u} & =R_{0}(z, \zeta) u+\left|\phi_{D}^{i}(z)\right\rangle r_{D}^{i}(z, \zeta) \tau, \\
G_{\lambda}(z, \zeta) \tilde{u} & =G_{0}(z, \zeta) u+G_{D}^{i}(z, \zeta) \tau, \\
W_{\lambda}(z) \tilde{u} & =V(z) u+W_{D}^{i}(z) \tau .
\end{aligned}
$$

\section{Resolvent Equations}

The basic resolvent equation utilized here is the Weinberg-van Winter equation cf. [16]. The $l$-connected and the $r$-connected parts of the resolvent, $I_{l}(z, \zeta)$ and $I_{r}(z, \zeta)$, are defined by

$$
\begin{aligned}
I_{l}(z, \zeta)= & \sum_{D_{n-1} \subset \ldots \subset D_{2}} \sum_{\left(\alpha_{1} \ldots \alpha_{n-1}\right)} R_{0}(z, \zeta) V_{\alpha_{1}}(z) \ldots \ldots \\
& \cdot R_{D_{n-1}}(z, \zeta) V_{\alpha_{2}}(z) \ldots R_{D_{2}}(z, \zeta) V_{\alpha_{n-1}}(z), \\
I_{r}(z, \zeta)= & \sum_{D_{n-1} \subset \ldots \subset D_{2}\left(\alpha_{1} \ldots \alpha_{n-1}\right)} V_{\alpha_{n-1}}(z) R_{D_{2}}(z, \zeta) . \\
& \cdot V_{\alpha_{2}}(z) R_{D_{n-1}}(z, \zeta) V_{\alpha_{1}}(z) R_{0}(z, \zeta),
\end{aligned}
$$

where $\sum_{D_{n-1} \subset \ldots \subset D_{2}}$ is over all chains of decompositions $D_{n-1} \subset \ldots \subset D_{2}$ such that $D_{j}$ arises from $D_{j+1}$ by joining two clusters of $D_{j+1}$, and $\sum_{\left(\alpha_{1} \ldots \alpha_{n-1}\right)}$ is over all $\left(\alpha_{1} \ldots \alpha_{n-1}\right)$ such that the particles of the pair $\alpha_{n-j}$ belong to the same cluster of $D_{j}$ but to different clusters of $D_{j+1}, j=2, \ldots, n-1$.

The disconnected part $D(z, \zeta)$ of the resolvent is given by

$$
D(z, \zeta)=\sum_{k \geqq 2} \sum_{D_{k}}(-1)^{k-1}(k-1) ! R_{D_{k}}(z, \zeta) .
$$

We then have the following two forms of the Weinberg-van Winter equation.

$$
\begin{aligned}
& R(z, \zeta)=I_{l}(z, \zeta) R(z, \zeta)+D(z, \zeta) \\
& R(z, \zeta)=R(z, \zeta) I_{r}(z, \zeta)+D(z, \zeta) .
\end{aligned}
$$

Equation (2.4) is given in [32], II, (6.38), and (2.5) is obtained from (2.4) by taking adjoints and replacing $(z, \zeta)$ by $(\bar{z}, \bar{\zeta})$. Note that

$$
I_{r}(z, \zeta)=I_{l}^{*}(\bar{z}, \bar{\zeta}), \quad D(z, \zeta)=D^{*}(\bar{z}, \bar{\zeta}) .
$$

In order to study the connected kernels we make the following 
Induction Assumption

$$
\sigma_{e}\left(H^{C}(z)\right) \cong \Sigma^{C}(\varphi)+e^{2 i \varphi} \overline{\mathrm{R}}^{+}
$$

for all systems $C$ with less than $n$ particles, where $H^{C}(z)$ is considered as an operator in $H^{t, l}$ for $t= \pm s, l=0,-2$.

Lemma 2.1. $\sigma\left(H_{D_{k}}(z)\right) \cong \sigma\left(H^{C_{1}}(z)\right)+\ldots+\sigma\left(H^{C_{k}}(z)\right)+e^{2 i \varphi} \overline{\mathrm{R}}^{+}$and

$$
\begin{aligned}
R_{D_{k}}(z, \zeta) \in & \bigcap_{\substack{t= \pm s \\
l=0,-2}} \mathscr{B}\left(H^{t, l}, H^{t, l+2}\right) \cap \mathscr{B}\left(H_{p_{D}}^{-s,-2} \otimes H_{k_{D}}^{s,-2}, H_{p_{D}}^{-s} \otimes H_{k_{D}}^{s}\right) \\
& \cap \mathscr{B}\left(H_{p_{D}}^{-s} \otimes H_{k_{D}}^{s}, H_{p_{D}}^{-s, 2} \otimes H_{k_{D}}^{s, 2}\right) \cap \mathscr{B}\left(L_{p_{D}}^{2} \otimes H_{k_{D}}^{s}, H_{p_{D}}^{0,2} \otimes H_{k_{D}}^{s, 2}\right)
\end{aligned}
$$

with $H_{D_{k}}$ considered as an operator in $H^{t, l}, t= \pm s, l=0,-2, H_{p_{D}}^{s,-2} \otimes H^{-s,-2}$, $H_{p_{D}}^{-s} \otimes H_{k_{D}}^{s}$ and $L_{p_{D}}^{2} \otimes H_{k_{D}}^{s}$ respectively, and $D \supset D_{k}$.

Proof. Let us consider the space $H_{p_{D}}^{s} \otimes H_{k_{D}}^{-s}$, the other cases are similar.

Let $\tilde{H}_{0}(z)$ be the closed operator in $H_{p_{D}}^{s} \otimes H_{k_{D}}^{-s}$ defined as $H_{0}(z)$, but with domain $H_{p_{D}}^{s, 2} \otimes H_{k_{D}}^{-s, 2}$. From the assumptions A1 and A2 it follows that $V_{\alpha}(z)$ is $H_{0, \alpha}-\varepsilon$-bounded, where $H_{0, \alpha}$ is the operator $z^{2} k_{\alpha}^{2} / 2 m_{\alpha}$ in $H_{k_{\alpha}}^{-s}$ with domain $H_{k_{\alpha}}^{-s, 2}$. This implies that $V_{\alpha}(z)$ is $\tilde{H}_{0}-\varepsilon$-bounded, and hence

$$
\left\|V_{D_{k}}(z) f\right\|_{s,-s}<\varepsilon\left\|\tilde{H}_{0} f\right\|_{s,-s}+K(\varepsilon)\|f\|_{s,-s}
$$

where $\|\cdot\|_{s,-s}=\|\cdot\|_{H_{p_{D}}^{-s} \otimes H_{k_{D}}^{s}}$.

From (2.6) follows that $H_{D_{k}}(z)$ as an operator in $H_{p_{D}}^{-s} \otimes H_{k_{D}}^{s}$ is closed on $\mathscr{D}\left(\tilde{H}_{0}\right)$, and the $H_{D_{k}}(z)$-norm is equivalent to the $\tilde{H}_{0}$-norm.

Moreover, the $\varepsilon$-boundedness implies that $H^{C}(z)$ is sectorial with arbitrarily small opening angle (see [29]) where $H^{C}(z)$ is considered as an operator in $H_{k_{C}}^{s}$ with domain $H_{k_{C}}^{s, 2}$. Then we can apply Ichinose's lemma [17] and the induction assumption to obtain

$$
\sigma\left(H_{D_{k}}(z)\right) \cong \sigma\left(H^{C_{1}}(z)\right)+\ldots+\sigma\left(H^{C_{1}}(z)\right)+e^{2 i \varphi} \overline{\mathrm{R}}^{+}
$$

and hence for $\zeta$ in the complement of this set

$$
R_{D_{k}}(z) \in \mathscr{B}\left(H_{p_{D}}^{-s} \otimes H_{k_{D}}^{s}, H_{p_{D}}^{-s, 2} \otimes H_{k_{D}}^{s, 2}\right) .
$$

Lemma 2.2. For $2 \leqq k \leqq n-2, \operatorname{Im} \zeta \neq 0$,

$$
\begin{gathered}
V_{\alpha_{1}} R_{0}(\zeta) \in \mathscr{C}\left(H_{\alpha_{1}}^{-s,-2}, H_{\alpha_{1}}^{s,-2}\right) \otimes \mathscr{B}\left(H_{M_{1}, \alpha_{2}}^{-s,-2}\right) \otimes \ldots \\
\otimes \mathscr{B}\left(H_{M_{k-1}, \alpha_{k}}^{-s,-2}\right) \otimes \mathscr{B}\left(H_{M_{k}, \alpha_{k+1}}^{s,-2}\right) \otimes \ldots \otimes \mathscr{B}\left(H_{M_{n-2}, \alpha_{n-1}}^{s,-2}\right),
\end{gathered}
$$

where $\left(\alpha_{1}, \ldots, \alpha_{n-1}\right)$ defines a connected graph, and $H_{M_{1-1}, \alpha_{i}}^{ \pm s,-2}$ are the spaces defined by (1.1) and (1.2) with $L^{2}$ replaced by $H^{ \pm s,-2}$.

Proof. We follow the proof of [10], using the representation

$$
\begin{aligned}
R_{0}(\zeta) & =\int_{0}^{\infty} e^{\zeta t} e^{-H_{0} t} \quad \alpha \\
& =\int_{0}^{\infty} e^{\zeta t} e^{-H_{0}^{\alpha_{1} t}} e^{-H^{M_{1}, \alpha_{2} t}} \cdot \ldots \cdot e^{-H_{0}^{M_{n-2}, \alpha_{n-1} d t}}
\end{aligned}
$$


valid for $\operatorname{Im} \zeta<0$ in the sense of uniform convergence in

$$
\begin{gathered}
\mathscr{B}\left(H_{\alpha_{1}}^{-s,-2}, H_{\alpha_{1}}^{-s}\right) \otimes \mathscr{B}\left(H_{M_{1}, \alpha_{2}}^{-s,-2}\right) \otimes \ldots \otimes \mathscr{B}\left(H_{M_{k-1}-\alpha_{k}}^{-s,-2}\right) \otimes \mathscr{B}\left(H_{M_{k}, \alpha_{k+1}}^{s,-2}\right) \\
\otimes \ldots \otimes \mathscr{B}\left(H_{M_{n-2}, \alpha_{n-1}}^{s,-2}\right) .
\end{gathered}
$$

Moreover, $e^{-H_{0}^{\alpha_{1} t}}$ is uniformly bounded in $\mathscr{B}\left(H_{\alpha_{1}}^{-s,-2}, H_{\alpha_{1}}^{-s}\right)$ and hence by A1 $V_{\alpha_{1}} e^{-H_{0}^{\alpha_{1} t}}$ uniformly bounded in $\mathscr{C}\left(H_{\alpha_{1}}^{-s,-2}, H_{\alpha_{1}}^{s,-2}\right)$ for $t \in[\varepsilon, \infty), \varepsilon>0$. Then the argument of [10] yields

$$
V_{\alpha_{1}} R_{0}(\zeta)=\int_{0}^{\infty} e^{\zeta t} V_{\alpha_{1}} e^{-H_{0}^{\alpha_{1} t}} e^{-H_{0}^{M_{1}}, \alpha_{2 t}} \cdot \ldots \cdot e^{-H_{0}^{M_{n-2}, \alpha_{n-1} t}} d t
$$

in the sense of uniform convergence in

$$
\begin{aligned}
\mathscr{C}\left(H_{\alpha_{1}}^{-s,-2}, H_{\alpha_{1}}^{s,-2}\right) & \otimes \mathscr{B}\left(H_{M_{1}, \alpha_{2}}^{-s,-2}\right) \otimes \ldots \otimes \mathscr{B}\left(H_{M_{k-1}, \alpha_{k}}^{-s,-2}\right) \\
& \otimes \mathscr{B}\left(H_{M_{k}, \alpha_{k+1}}^{s,-2}\right) \otimes \ldots \otimes \mathscr{B}\left(H_{M_{n-2}, \alpha_{k+1}}^{s,-2}\right)
\end{aligned}
$$

and the lemma follows.

Lemma 2.3. Let $\left(\alpha_{1}, \ldots, \alpha_{n-1}\right)$ define a connected graph, and $V_{D_{n-\imath}}=\sum_{j=1}^{i} V_{\alpha_{j}}$. For $2 \leqq i \leqq n-2, \operatorname{Im} \zeta \neq 0$,

$$
V_{D_{n-i}} R_{D_{n-i}}(\zeta) \in \mathscr{B}\left(H_{k_{D_{n-1}}}^{s,-2} \otimes \mathscr{B}\left(H_{M_{i}, \alpha_{i+1}}^{-s,-2}\right) \otimes \ldots \otimes \mathscr{B}\left(H_{M_{n-2}, \alpha_{n-1}}^{-s,-2}\right) .\right.
$$

Proof. We use the representation

$$
V_{D_{n-i}} R_{D_{n-i}}(\zeta)=\int_{0}^{\infty} e^{\zeta t} V_{D_{n-i}} e^{-H_{D_{n-i}}^{\prime} t} e^{-H_{0}^{M_{i}}, \alpha_{i}+1 t} \cdot \ldots \cdot e^{-H_{0}^{M_{n-2}, \alpha_{n-1} t}},
$$

where $H_{D_{n-\imath}}^{\prime}=H_{0}^{M_{i}}+V_{D_{n-i}}$, valid for $\operatorname{Im} \zeta<0$ in the sense of uniform convergence in

$$
\mathscr{B}\left(H_{k_{D_{n-i}}}^{s,-2} \otimes \mathscr{B}\left(H_{M_{i}, \alpha_{i+1}}^{-s,-2}\right) \otimes \ldots \otimes \mathscr{B}\left(H_{M_{n-2}, \alpha_{n-1}}^{-s,-2}\right)\right.
$$

by Lemma 2.1 and the same proof as in Lemma 2.2.

Lemma 2.4. $I_{r}(\zeta) \in \mathscr{C}\left(H^{-s,-2}, H^{s,-2}\right)$ for $\operatorname{Im} \zeta \neq 0$.

Proof. A typical term is

$$
V_{\alpha_{n-1}} R_{D_{2}}(\zeta) \cdot \ldots \cdot V_{\alpha_{k+1}} R_{D_{n-k}}(\zeta) \cdot \ldots \cdot V_{\alpha_{1}} R_{0}(\zeta)
$$

We prove by induction that for $i=1, \ldots, n-1$

$$
\begin{gathered}
V_{\alpha_{l}} R_{D_{n-1+1}}(\zeta) \cdot \ldots \cdot V_{\alpha_{1}} R_{0}(\zeta) \in \mathscr{C}\left(H_{k_{D_{n-l}}}^{-s,-2} H_{D_{n-i}}^{s,-2}\right) \\
\otimes \mathscr{B}\left(H_{M_{i}, \alpha_{i+1}}^{-s,-2}\right) \otimes \ldots \otimes \mathscr{B}\left(H_{M_{n-2}, \alpha_{n-1}}^{s,-2}\right) .
\end{gathered}
$$

For $i=1$ (2.12) holds by Lemma 2.2. Compactness of the term given by (2.11) is (2.12) for $i=n-1$.

Assuming now (2.12) for $i=k$, we shall prove (2.12) for $i=k+1$. We write

$$
V_{\alpha_{k+1}} R_{D_{n-k}}(\zeta)=V_{\alpha_{k+1}} R_{0}(\zeta)\left(1-V_{D_{n-k}} R_{D_{n-k}}(\zeta)\right) \text {. }
$$


By (2.12) for $i=k$ and Lemma 2.3 we get

$$
\begin{aligned}
& \left(1-V_{D_{n-k}} R_{D_{n-k}}(\zeta)\right) V_{\alpha_{k}} R_{D_{n-k+1}}(\zeta) \cdot \ldots \cdot V_{\alpha_{1}} R_{0}(\zeta) \\
& \quad \in \mathscr{C}\left(H_{k_{D_{n-k}}-s,-2}^{-2} H_{k_{D_{n-k}}}^{s,-2}\right) \otimes \mathscr{B}\left(H_{M_{k}, \alpha_{k}+1}^{-s,-2}\right) \otimes \ldots \otimes \mathscr{B}\left(H_{M_{n-2}, \alpha_{n-1}}^{-s,-2}\right)
\end{aligned}
$$

Lemma 2.2 implies

$$
\begin{aligned}
V_{\alpha_{k+1}} R_{0}(\zeta) \in & \mathscr{B}\left(H_{k_{D_{n-k}}}^{s,-2}\right) \otimes \mathscr{C}\left(H_{\alpha_{k+1}}^{-s,-2}, H_{\alpha_{k+1}}^{s,-2}\right) \\
& \otimes \mathscr{B}\left(H_{M_{k+1}, \alpha_{k+2}}^{-s,-2}\right) \otimes \ldots \otimes \mathscr{B}\left(H_{M_{n-2}, \alpha_{n-1}}^{-s,-2}\right),
\end{aligned}
$$

where $k_{D_{n-k}}^{\prime}$ is the set of internal momentum vectors of particles in clusters of $D_{n-k}$ with exception of a possible common particle with $\alpha_{k+1}$ together with the relative momenta of centers of mass of clusters of $D_{n-k}$ and $\alpha_{k+1}$.

From (2.13)-(2.15) follows (2.12) for $i=k+1$, and the lemma is proved.

Corollary 2.5. $I_{l}(\zeta) \in \mathscr{C}\left(H^{-s, 2}, H^{s, 2}\right)$ for $\operatorname{Im} \zeta \neq 0$.

Lemma 2.6. $I_{r}(\zeta) \in \mathscr{C}\left(H^{-s}, H^{s}\right)$ for $\operatorname{Im} \zeta \neq 0$.

Proof. This is proved in the same way as Lemma 2.4 on replacing -2 by 0 and 0 by 2 in Lemmas 2.2-2.4.

Lemma 2.7. For $-a<\varphi<a, \zeta \notin \Sigma(\varphi)+e^{2 i \varphi} \overline{\mathrm{R}}^{+}$

$$
\begin{aligned}
& I_{r}(z, \zeta) \in \mathscr{C}\left(H^{-s,-2}, H^{s,-2}\right) \cap \mathscr{C}\left(H^{-s}, H^{s}\right) \\
& I_{l}(z, \zeta) \in \mathscr{C}\left(H^{-s, 2}, H^{s, 2}\right) \cap \mathscr{C}\left(H^{-s}, H^{s}\right),
\end{aligned}
$$

and the functions $I_{r}(z, \zeta)$ and $I_{l}(z, \zeta)$ are analytic in $z$ and $\zeta$.

Proof. By successive applications of Lemma 2.1 and A1, A2 it is proved that

$$
I_{r}(z, \zeta) \in \mathscr{B}\left(H^{-s,-2}, H^{s,-2}\right) \cap \mathscr{B}\left(H^{-s}, H^{s}\right) .
$$

Then compactness follows from Lemmas 2.4 and 2.6 and Corollary 2.5 by analytic continuation.

Lemma 2.8. $\sigma_{e}(H(z)) \cong \Sigma(\varphi)+e^{2 i \varphi} \overline{\mathrm{R}}^{+}$, where $H(z)$ is considered as an operator in $H^{t, l}$ for $t= \pm s, l=0,-2$.

Proof. It follows from Lemma 2.1 and an $\varepsilon$-boundedness argument, that

$$
\left\|I_{r}(z, \zeta)\right\| \rightarrow 0 \quad \text { for } \quad|\zeta| \rightarrow \infty, \quad\left|\operatorname{Arg} \zeta-e^{2 i \varphi}\right| \geqq \delta>0,
$$

in any of the spaces $\mathscr{B}\left(H^{t, l}\right), t= \pm s, l=-2,0$.

Then by Lemma 2.7 and the analytic Fredholm theory (cf. [31]), $\left(1-I_{r}(z, \zeta)\right)^{-1}$ is meromorphic in $\mathrm{C} \backslash\left\{\Sigma(\varphi)+e^{2 i \varphi} \overline{\mathrm{R}}^{+}\right\}$with values in $\left(H^{t, l}\right)$. It then follows from (2.5) and Lemma 2.1 that

$$
R(z, \zeta)=D(z, \zeta)\left(1-I_{r}(z, \zeta)\right)^{-1}
$$

is meromorphic in $C \backslash\left\{\Sigma(\varphi)+e^{2 i \varphi} \overline{\mathrm{R}}^{+}\right\}$with values in $\mathscr{B}\left(H^{t, l}\right)$, and the lemma is proved.

In the proof we have used the induction assumption which by the lemma is verified for systems of $n$ particles. The verification for 2-body systems is straightforward. 
Lemma 2.9. The operator $1-I_{l}(z, \zeta)$ has the same null space when considered as an operator in $H^{t, l}$ for $t= \pm s, 0$ and $l=0,2$. The same holds true of the operator $H(z)-\zeta$, and $\sigma_{d}(H(z))$ is the same when $H(z)$ is considered as an operator in $H^{t, l}$ for $t= \pm s, 0$ and $l=0,2$.

Proof. For $1-I_{l}(z, \zeta)$ this follows from Lemma 2.7. Moreover,

$$
1-I_{l}(z, \zeta)=D(z, \zeta)(H(z)-\zeta)
$$

and hence

$$
\mathscr{N}(H(z)-\zeta) \subseteq \mathscr{N}\left(1-I_{l}(z, \zeta)\right) .
$$

We recall the basic property of discrete eigenvalues and eigenfunctions of $H(z)$.

Lemma 2.10. 1) $\sigma_{d}^{r}(\varphi)=\sigma_{d}(H(z)) \cap \mathrm{R}=\sigma_{p}(H) \backslash \Sigma$, and $\sigma_{d}^{c}(\varphi)=\sigma_{d}(H(z)) \backslash \mathrm{R}$ accumulates at most at points of $\Sigma(\varphi)$, is contained in the sector bounded by the half-lines $\Sigma_{2}^{0}+\mathrm{R}^{+}$and $\Sigma_{2}^{0}+e^{2 i \varphi} \mathrm{R}^{+}$, and is independent of $\varphi$ unless "absorbed" in the essential spectrum $\Sigma(\varphi)+e^{2 i \varphi} \mathrm{R}^{+}$.

2) For $\lambda \in \sigma_{d}(\varphi)$ there exists a basis $\left\{\phi_{\lambda}^{i}(z)\right\}_{i=1}^{d_{\lambda}}$ of $\mathscr{N}(H(z)-\lambda)$, such that the vector-valued functions $\phi_{\lambda}^{i}(z)$ are analytic in $\mathcal{O}$ for $\lambda \in \sigma_{d}^{r}(\varphi)$ and analytic in $\left\{z=\varrho e^{i \varphi} \mid \varrho>0, \varphi \gtrless \operatorname{Arg} \lambda\right.$ if $\left.\operatorname{Arg} \lambda \gtrless 0\right\}, i=1 \ldots d_{\lambda}=\operatorname{dim} \mathscr{N}(H(z)-\lambda)$.

3) For $\lambda \in \sigma_{d}(\varphi)$ the inverse Fourier transforms $\tilde{\phi}_{\lambda}^{i}(z)$ of the eigenfunctions are of the form

$$
\tilde{\phi}_{\lambda}^{i}(z)=e^{-k|x|} g, \quad g \in \mathscr{H},
$$

where $k$ depends on $\lambda$ and $z$.

Proof. 1) and 2) are proved in [2] and [4], and 3) in [11].

Definition 2.11. For $\lambda \in \Sigma$ we define the set of resonances $\mathscr{R}_{\lambda}$ by

$$
\mathscr{R}_{\lambda}=\bigcup_{-a<\varphi<0}\left\{\kappa \in \sigma_{d}^{c}(\varphi) \mid \kappa \text { is between }\left\{\lambda+e^{2 i \varphi} \overline{\mathrm{R}}^{+}\right\} \text {and }\left\{\lambda^{\prime}+e^{2 i \varphi} \overline{\mathrm{R}}^{+}\right\}\right\} \text {, }
$$

where $\lambda^{\prime}=\min \{\mu \in \Sigma \mid \mu>\lambda\}$.

We set $\mathscr{R}_{\lambda^{\prime}}^{\prime}=\mathscr{R}_{\lambda}$.

We have

$$
\overline{\mathscr{R}}_{\lambda}=\bigcup_{0<\varphi<a}\left\{\kappa \in \sigma_{d}^{c}(\varphi) \mid \kappa \text { is between }\left\{\lambda+e^{2 i \varphi} \overline{\mathrm{R}}^{+}\right\} \text {and }\left\{\lambda^{\prime}+e^{2 i \varphi} \overline{\mathrm{R}}^{+}\right\}\right\} .
$$

For $\varphi \in(-a, a)$ we set

$$
\tilde{\sigma}_{d}(\varphi)=\left\{\kappa \in C \backslash \sigma_{e}(\varphi) \mid \mathcal{N}\left(1-I_{r}(z, \kappa)\right) \neq\{0\}\right\},
$$

and for $\varphi \neq 0$

$$
\tilde{\sigma}_{d}^{r}(\varphi)=\tilde{\sigma}_{d}(\varphi) \cap \mathrm{R}=\tilde{\sigma}_{p}, \quad \tilde{\sigma}_{d}^{c}(\varphi)=\tilde{\sigma}_{d}(\varphi) \backslash \mathrm{R} .
$$

By analyticity and unitary equivalence, $\tilde{\sigma}_{d}(\varphi)$ is $\varphi$-independent in the same way as $\sigma_{d}(\varphi)$. We define

$$
\begin{aligned}
& \tilde{\mathscr{R}}_{\lambda}=\bigcup_{-a<\varphi<0}\left\{\kappa \in \tilde{\sigma}_{d}^{c}(\varphi) \mid \kappa \text { is between }\left\{\lambda+e^{2 i \varphi} \overline{\mathrm{R}}^{+}\right\} \text {and }\left\{\lambda^{\prime}+e^{2 i \varphi} \overline{\mathrm{R}}^{+}\right\}\right\}, \\
& \tilde{\mathscr{R}}_{\lambda^{\prime}}^{\prime}=\tilde{\mathscr{R}}_{\lambda} .
\end{aligned}
$$


We shall now derive a decomposition equation for the resolvent based on the Weinberg-van Winter equation in the form (2.16), valid in $\mathscr{B}\left(H^{t, l}\right)$ for $t= \pm s, 0$, $l=0,-2$.

We decompose $D(z, \zeta)$ corresponding to a fixed energy $E$ by splitting off from each operator $R_{D}(z, \zeta)$ the parts arising from bound states of the clusters $C_{i}$ of $D$ with total energy less than $E$. Using the notation of Sect. 1 , by $(1.21)$ and the $2^{\text {nd }}$ resolvent equation we have for $D \in \mathscr{D}_{E}$

$$
\begin{aligned}
R_{D}(z, \zeta)= & \sum_{i=1}^{n(D)}\left|\phi_{D}^{i}(z)\right\rangle r_{D}^{i}(z, \zeta)\left\langle\phi_{D}^{i}(\bar{z})\right| \\
& +R_{0}(z, \zeta)\left(1-P_{D}^{E}(z)-V_{D}(z) R_{D}^{E}(z, \zeta)\right)
\end{aligned}
$$

and for $D_{k} \in \mathscr{D}_{E}^{\prime}, 2 \leqq k \leqq n-1$

$$
R_{D_{k}}(z, \zeta)=R_{0}(z, \zeta)\left(1-V_{D_{k}}(z) R_{D_{k}}(z, \zeta)\right) .
$$

Introducing (2.19) and (2.20) in (2.3) and using the notation (1.23), (1.24), we obtain from (2.16) the following representation of $R(z, \zeta)$.

Lemma 2.12. For $\zeta \in C \backslash\left\{\left(\Sigma(\varphi)+e^{2 i \varphi} \overline{\mathrm{R}}^{+}\right) \cup \tilde{\sigma}_{d}(\varphi)\right\}$

$$
R(z, \zeta)=J(z) R_{1}(z, \zeta) Y(z, \zeta),
$$

where

$$
Y(z, \zeta)=X(z, \zeta)\left(1-I_{r}(z, \zeta)\right)^{-1}
$$

and

$$
X(z, \zeta)=\left(X_{E}(z, \zeta),\left\{X_{D}^{i}(z, \zeta)\right\}\right)
$$

with

$$
\begin{aligned}
X_{E}(z, \zeta)= & -\sum_{D \in \mathscr{D}_{E}}\left(1-P_{D^{E}}^{E}(z)-V_{D}(z) R_{D}^{E}(z, \zeta)\right) \\
& +\sum_{D_{k} \in \mathscr{D}_{E}^{\prime}}(-1)^{k-1}(k-1) !\left(1-V_{D_{k}}(z) R_{D_{k}}(z, \zeta)\right)
\end{aligned}
$$

and

$$
X_{D}^{i}(z, \zeta)=-\left\langle\phi_{D}^{i}(\bar{z})\right|, \quad D \in \mathscr{D}_{E}, \quad i=1 \ldots n(D) .
$$

We have the following identities

\section{Lemma 2.13.}

$$
\begin{array}{ll}
R(z, \zeta) G(z, \zeta)=J(z) R_{1}(z, \zeta) \quad \text { in } \mathscr{B}\left(\tilde{H}^{s}, H^{s, 2}\right), \\
G(z, \zeta) Y(z, \zeta)=I & \text { in } \mathscr{B}\left(H^{s}\right) .
\end{array}
$$

Proof. By Lemmas 2.1 and 2.6 and the proof of Lemma 2.8

$$
X(z, \zeta) \in \mathscr{B}\left(H^{s}, \tilde{H}^{s}\right), \quad Y(z, \zeta) \in \mathscr{B}\left(H^{s}, \tilde{H}^{s}\right) .
$$

From A1 and A2 follows that $G(z, \zeta)$ defined by (1.29) satisfies

$$
G(z, \zeta) \in \mathscr{B}\left(\tilde{H}^{s}, H^{s}\right) \text {. }
$$


We prove (2.26), then (2.27) follows from (2.26) and Lemma 2.12. By the second resolvent equation

$$
R(z, \zeta) G_{0}(z, \zeta)=R_{0}(z, \zeta)
$$

and

$$
\begin{aligned}
& R(z, \zeta) W_{D}^{i}(z) r_{D}^{i}(z, \zeta) \\
& \quad=R(z, \zeta) V_{D}^{\prime}(z) R_{D}(z, \zeta)\left(H_{D}(z)-\zeta\right)\left|\phi_{D}^{i}(z)\right\rangle r_{D}^{i}(z, \zeta) \\
& \quad=\left|\phi_{D}^{i}(z)\right\rangle r_{D}^{i}(z, \zeta)-R(z, \zeta)\left|\phi_{D}^{i}(z)\right\rangle,
\end{aligned}
$$

hence

$$
R(z, \zeta) G_{D}^{i}(z, \zeta)=\left|\phi_{D}^{i}(z)\right\rangle r_{D}^{i}(z, \zeta)
$$

and (2.26) follows from (2.28), (2.29).

For $\varphi \neq 0$ we obtain, starting from (1.30), the following alternative decomposition of $R(z, \zeta)$, proved in the same way as Lemma 2.12.

Lemma 2.14. For $\varphi \neq 0, \lambda=\lambda_{D}^{i} \in \sigma_{d}^{D}, \zeta \in C \backslash\left\{\left(\Sigma(\varphi)+e^{2 i \varphi} \overline{\mathrm{R}}^{+}\right) \cup \tilde{\sigma}_{d}(\varphi)\right\}$

$$
R(z, \zeta)=J_{\lambda}(z) R_{1, \lambda}(z, \zeta) Y_{\lambda}(z, \zeta),
$$

where

$$
Y_{\lambda}(z, \zeta)=X_{\lambda}(z, \zeta)\left(1-I_{r}(z, \zeta)\right)^{-1}=\left(Y_{\lambda}^{0}(z, \zeta), Y_{D}^{i}(z, \zeta)\right)
$$

and

$$
X_{\lambda}(z, \zeta)=\left(X_{\lambda}^{0}(z, \zeta), X_{D}^{i}(z, \zeta)\right)
$$

with

$$
\begin{aligned}
X_{\lambda}^{0}(z, \zeta)= & -\left(1-P_{D}^{i}(z)-V_{D}(z) R_{D}^{\lambda}(z, \zeta)\right) \\
& +\sum_{D_{k} \in \mathscr{D} \dot{\lambda}}(-1)^{k-1}(k-1) !\left(1-V_{D_{k}}(z) R_{D_{k}}(z, \zeta)\right)
\end{aligned}
$$

and

$$
X_{D}^{i}(z, \zeta)=-\left\langle\phi_{D}^{i}(\bar{z})\right|
$$

where

$$
\mathscr{D}_{\lambda}^{\prime}=\left\{D_{2} \neq D\right\} \cup\left\{D_{k} \mid k \geqq 3\right\}
$$

We have

$$
X_{\lambda}(z, \zeta) \in \mathscr{B}\left(H^{s}, \tilde{H}_{\lambda}^{s}\right), \quad Y_{\lambda}(z, \zeta) \in \mathscr{B}\left(H^{s}, \tilde{H}_{\lambda}^{s}\right), \quad G_{\lambda}(z, \zeta) \in \mathscr{B}\left(\tilde{H}_{\lambda}^{s}, H^{s}\right)
$$

and the following identities, proved as Lemma 2.13.

\section{Lemma 2.15.}

$$
\begin{array}{ll}
R(z, \zeta) G_{\lambda}(z, \zeta)=J_{\lambda}(z) R_{1, \lambda}(z, \zeta) & \text { in } \quad \mathscr{B}\left(\tilde{H}_{\lambda}^{s}, H^{s, 2}\right), \\
G_{\lambda}(z, \zeta) Y_{\lambda}(z, \zeta)=I & \text { in } \quad \mathscr{B}\left(H^{s}\right) .
\end{array}
$$




\section{Limiting Absorption Principle}

In this section we prove the existence of the limits on the continuous spectrum of the operators $I_{r}(z, \zeta), I_{l}(z, \zeta), Y(z, \zeta)$, and $G(z, \zeta)$ in topologies related to the $H^{s}$-spaces and establish the analyticity properties of the limiting operators.

The statement

$$
Q_{ \pm}\left(z, \lambda_{D}^{i}+\mu e^{2 i \varphi}\right) \in \mathscr{B}\left(K_{1}, K_{2}\right)
$$

means that the following limits exist in the uniform operator topology of $\mathscr{B}\left(K_{1}, K_{2}\right)$

$$
\begin{aligned}
Q_{ \pm}\left(z, \lambda_{D}^{i}+\mu e^{2 i \varphi}\right)= & \lim _{\varepsilon \downarrow 0} Q\left(z, \lambda_{D}^{i}+\mu e^{2 i(\varphi \pm \varepsilon)}\right) \\
& =\lim _{\varepsilon \downarrow 0} Q\left(\varrho e^{i(\varphi \mp \varepsilon)}, \lambda_{D}^{i}+\mu e^{2 i \varphi}\right) .
\end{aligned}
$$

Lemma 3.1. For $D=\left\{C_{1}, C_{2}\right\}$ and $\alpha \cap C_{j} \neq \emptyset, j=1,2$,

$$
V_{\alpha}(z) \in \mathscr{B}\left(H_{p_{D}}^{-s, 2} \otimes H_{k_{D}}^{s, 2}, H_{p_{\alpha}}^{s} \otimes H_{k_{D}}^{s, 2}\right) .
$$

Proof. It is straightforward to verify that

$$
A_{\alpha}(z) \in \mathscr{B}\left(H_{p_{D}}^{-s, 2} \otimes H_{k_{D}}^{s, 2}, H_{p_{\alpha}}^{0,2} \otimes H_{k_{D}}^{s, 2}\right),
$$

and the lemma follows from $\mathrm{A} 1$ and $\mathrm{A} 2$.

Lemma 3.2. For $D=\left\{C_{1}, C_{2}\right\}, \alpha \cap C_{j} \neq \emptyset, j=1,2$ and $i=1 \ldots n(D)$,

$$
\left|\phi_{D}^{i}(z)\right\rangle r_{D_{ \pm}}^{i}\left(z, \lambda_{D}^{i}+\mu e^{2 i \varphi}\right)\left\langle\phi_{D}^{i}(\bar{z})\right| V_{\alpha}(z) \in \mathscr{B}\left(H_{p_{D}}^{-s, 2} \otimes H_{k_{D}}^{s, 2}\right),
$$

where

$$
\begin{aligned}
r_{D_{ \pm}}^{i}\left(z, \lambda_{D}^{i}+\mu e^{2 i \varphi}\right) & =\lim _{\varepsilon \downarrow 0} r_{D}^{i}\left(z, \lambda_{D}^{i}+\mu e^{2 i(\varphi \pm \varepsilon)}\right) \\
& =\lim _{\varepsilon \downarrow 0} r_{D}^{i}\left(\varrho e^{i(\varphi \mp \varepsilon)}, \lambda_{D}^{i}+\mu e^{2 i \varphi}\right) .
\end{aligned}
$$

Proof. This follows from Lemmas 2.10(3) and 3.1 together with a result of Agmon [1], quoted in [8], Lemma 1.1.

Lemma 3.3. For $D=\left\{C_{1}, C_{2}\right\}, \alpha \cap C_{j} \neq \emptyset, j=1,2, i=1, \ldots, n(D), \varphi=0, \mu \in\left(0, E-\lambda_{D}^{i}\right)$ and $\varphi \neq 0, \mu \in \mathrm{R}^{+}$

$$
R_{D}^{E}\left(z, \lambda_{D}^{i}+\mu e^{2 i \varphi}\right) V_{\alpha}(z) \in \mathscr{B}\left(H_{p_{D}}^{-s, 2} \otimes H_{k_{D}}^{s, 2}, H^{s, 2}\right) .
$$

For $\varphi \neq 0, \lambda=\lambda_{D}^{i}$, the same holds with $R_{D}^{E}$ replaced by $R_{D}^{\lambda}$.

Proof. By Lemma 3.1 it suffices to prove $R_{D}^{E}\left(z, \lambda_{D}^{i}+\mu e^{2 i \varphi}\right) \in \mathscr{B}\left(H^{s}, H^{s, 2}\right)$. This is proved in the same way as Lemma 2.1. Defining $H_{0}(z)$ as a closed operator in $H^{s}$ with domain $H^{s, 2}$ and accordingly by $\varepsilon$-boundedness $H_{D}(z)$ closed on $H^{s, 2}$, we obtain

$$
H_{D}^{E}(z)=H_{D}(z)\left(1-p_{D}^{E}(z)\right) H^{s}
$$

as a closed operator in $\left(1-P_{D}^{E}(z)\right) H^{s}$ with domain $\left.\left(1-P_{D}^{E}\right)\right) H^{s, 2}$ and graph-norm equivalent with the $H^{s, 2}$-norm. By the induction assumption and Ichinose's lemma, $\lambda_{D}^{i}+\mu e^{2 i \varphi}$ is in the resolvent set of $H_{D}^{E}(z)$ and hence

$$
R_{D}^{E}\left(z, \lambda_{D}^{i}+\mu e^{2 i \varphi}\right) \in \mathscr{B}\left(H^{s}, H^{s, 2}\right) .
$$


Lemma 3.4. For $\varphi \neq 0, \mu \in \mathrm{R}^{+}$

$$
I_{l \pm}\left(z, \lambda_{D}^{i}+\mu e^{2 i \varphi}\right) \in \mathscr{C}\left(H_{p_{D}}^{-s, 2} \otimes H_{k_{D}}^{s, 2}\right) .
$$

Proof. A typical term containing the singularity is

$$
R_{0}(z, \zeta) V_{\alpha_{1}}(z) \cdot \ldots \cdot R_{D_{n-k}}(z, \zeta) V_{\alpha_{k+1}}(z) \cdot \ldots \cdot R_{D}(z, \zeta) V_{\alpha_{n-1}}(z)
$$

By Lemmas 3.2 and 3.3

$$
R_{D \pm}\left(z, \lambda_{D}^{i}+\mu e^{2 i \varphi}\right) V_{\alpha_{n-1}}(z) \in \mathscr{B}\left(H_{p_{D}}^{-s, 2} \otimes H_{k_{D}}^{s, 2}\right) .
$$

The factors $R_{D_{n-k}}\left(z, \lambda_{D}^{i}+\mu e^{2 i \varphi}\right) V_{\alpha_{k+1}}(z), k=0, \ldots, n-3$, are in $\mathscr{B}\left(H_{p_{D}}^{-s, 2} \otimes H_{k_{D}}^{s, 2}\right)$ by Lemma 2.1 .

Hence the term given by (3.1) is in $\mathscr{B}\left(H_{p_{D}}^{-s, 2} \otimes H_{k_{D}}^{s, 2}\right)$.

The terms without singularity are in $\mathscr{B}\left(H_{p_{D}}^{-s, 2} \otimes H_{k_{D}}^{s, 2}\right)$ by repeated application of Lemma 2.1 and hence

$$
I_{l \pm}\left(z, \lambda_{D}^{i}+\mu e^{2 i \varphi}\right) \in \mathscr{B}\left(H_{p_{D}}^{-s, 2} \otimes H_{k_{D}}^{s, 2}\right) .
$$

By Lemma 2.6, $I_{l}\left(z, \lambda_{D}^{i}+\mu e^{2 i(\varphi \pm \varepsilon)}\right) \in \mathscr{C}\left(H_{p_{D}}^{-s, 2} \otimes H_{k_{D}}^{s, 2}\right)$ and the lemma follows.

Lemma 3.5. For $\varphi=0, \mu \in\left(\Sigma_{2}^{0}, E\right) \backslash \Sigma_{2}$

$$
I_{l \pm}(\mu) \in \mathscr{C}\left(H^{-s, 2}\right) .
$$

Proof. We consider the term

$$
R_{0}(\mu) V_{\alpha_{1}} \cdot \ldots \cdot R_{D_{n-k}}(\mu) V_{\alpha_{k+1}} \cdot \ldots \cdot R_{D \pm}(\mu) V_{\alpha_{n-1}},
$$

where

$$
R_{D \pm}(\mu)=\sum_{i=1}^{n(D)}\left|\phi_{D}^{i}\right\rangle r_{D \pm}^{i}(\mu)\left\langle\phi_{D}^{i}\right|+R_{D}^{E}(\mu) .
$$

Using A1 and Lemma 2.10(3), it is easy to show as in Lemma 3.1 that

$$
\left|\phi_{D}^{i}\right\rangle V_{\alpha_{n-1}} \in \mathscr{B}\left(H^{-s, 2}, H^{s}\right) \text {, }
$$

and hence by [8], Lemma 1.1

$$
\left|\phi_{D}^{i}\right\rangle r_{D \pm}^{i}(\mu)\left\langle\phi_{D}^{i}\right| V_{\alpha_{n-1}} \in \mathscr{B}\left(H^{-s, 2}\right) .
$$

By a similar proof as in Lemma 3.3

$$
R_{D}^{E}(\mu) V_{\alpha_{n-1}} \in \mathscr{B}\left(H^{-s, 2}\right) .
$$

The factors $R_{D_{n-k}}(\mu) V_{\alpha_{k+1}}$ are in $\mathscr{B}\left(H^{-s, 2}\right)$ for $k=0, \ldots, n-3$ by Lemma 2.1. Hence by (3.4) and (3.5), $I_{l \pm}(\mu) \in \mathscr{B}\left(H^{-s, 2}\right)$, and then by Corollary 2.5, $I_{l \pm}(\mu) \in \mathscr{C}\left(H^{-s, 2}\right)$.

Lemma 3.6. For $i=1 \ldots n(D), \varphi=0, \lambda_{D}^{i}+\mu \in\left(\Sigma_{2}^{0}, E\right) \backslash \Sigma_{2}$ and $\varphi \neq 0, \mu \in \mathrm{R}^{+}$

$$
I_{r \pm}\left(z, \lambda_{D}^{i}+\mu e^{2 i \varphi}\right) \in \mathscr{C}\left(H^{s}\right) .
$$

Proof. We consider the typical term, setting $\zeta=\lambda_{D}^{i}+\mu e^{2 i \varphi}$,

$$
V_{\alpha_{n-1}}(z) R_{D \pm}(z, \zeta) \cdot \ldots \cdot V_{\alpha_{k+1}}(z) R_{D_{n-k}}(z, \zeta) \cdot \ldots \cdot V_{\alpha_{1}}(z) R_{0}(z, \zeta)
$$


where

$$
V_{\alpha_{n-1}}(z) R_{D \pm}(z, \zeta)=\sum_{i=1}^{n(D)} V_{\alpha_{n-1}}(z)\left|\phi_{D}^{i}(z)\right\rangle r_{D \pm}^{i}(z, \zeta)\left\langle\phi_{D}^{i}(\bar{z})\right|+V_{\alpha_{n-1}}(z) R_{D}^{E}(z, \zeta) .
$$

By the proof of Lemma 3.3, $V_{\alpha_{n-1}}(z) R_{D}^{E}(z, \zeta) \in \mathscr{B}\left(H^{s}\right)$.

By Lemmas 2.10(3) and 3.1 and [8], Lemma 1.1

$$
V_{\alpha_{n-1}}(z)\left|\phi_{D}^{i}(z)\right\rangle r_{D \pm}^{i}(z, \zeta)\left\langle\phi_{D}^{i}(\bar{z})\right| \in \mathscr{B}\left(H^{s}\right) .
$$

Hence $I_{r \pm}(z, \zeta) \in \mathscr{B}\left(H^{s}\right)$, and then by Lemma $2.6 I_{r \pm}(z, \zeta) \in \mathscr{C}\left(H^{s}\right)$.

Lemma 3.7. For $\varphi \in(-a, a), \lambda \in \Sigma_{2}, \zeta=\lambda+\mu e^{2 i \varphi}, \mu \in \mathrm{R}^{+}$,

$$
G_{ \pm}(z, \zeta) \in \mathscr{B}\left(\tilde{H}^{s}, H^{s}\right), \quad G_{\lambda \pm}(z, \zeta) \in \mathscr{B}\left(\tilde{H}_{\lambda}^{s}, H^{s}\right) .
$$

Proof. Clearly $G_{0}(z, \zeta) \in \mathscr{B}\left(H^{s}\right)$, and by Lemma 2.1

$$
\begin{array}{rrrr}
G_{D}^{i}(z, \zeta) \in \mathscr{B}\left(H_{p_{D}}^{s}, H^{s}\right) & \text { for } & \varphi=0, & \lambda_{D}^{i}>\lambda \\
\text { and for } & \varphi \neq 0, & \lambda_{D}^{i} \neq \lambda .
\end{array}
$$

For $\varphi=0, \lambda_{D}^{i} \leqq \lambda$ and $\varphi \neq 0, \lambda_{D}^{i}=\lambda$ we have by Lemma 3.1 and [8], Lemma 1.1

$$
W_{D}^{i}(z, \zeta) r_{D \pm}^{i}(z, \zeta)=V_{D}^{\prime}(z)\left|\phi_{D}^{i}(z)\right\rangle r_{D \pm}^{i}(z, \zeta) \in \mathscr{B}\left(H_{p_{D}}^{s}, H^{s}\right),
$$

and the lemma is proved.

Lemma 3.8. For $\lambda=\lambda_{D}^{i}, \kappa=\lambda+\mu e^{2 i \varphi}, \varphi \neq 0$,

$$
\mathscr{N}\left(1-I_{l_{(-)}^{+}}(z, \kappa)\right) \neq\{0\}
$$

if and only if $\kappa \in \tilde{\mathscr{R}}_{\lambda} \cup \overline{\tilde{\mathscr{R}}}_{\lambda}^{\prime}\left(\tilde{\mathscr{R}}_{\lambda}^{\prime} \cup \overline{\mathscr{R}}_{\lambda}\right)$, where

$$
I_{l \pm}(z, \kappa) \in \mathscr{C}\left(H_{p_{D}}^{-s, 2} \otimes H_{k_{D}}^{s, 2}\right) .
$$

For $\varphi=0, \mathscr{N}\left(1-I_{l_{(-)}^{+}}(z, \kappa)\right) \neq\{0\}$ if and only if $\kappa \in \tilde{\sigma}_{p}$, where

$$
I_{l \pm}(z, \kappa) \in \mathscr{C}\left(H^{-s, 2}\right) .
$$

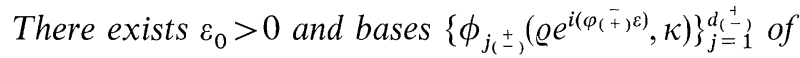

$$
\mathscr{N}\left(1-I_{l}\left(e^{i\left(\varphi_{(} \mp\right) \varepsilon}, \kappa\right)\right)
$$

and a basis $\left\{\phi_{j}(z, \kappa)\right\}_{j=1}^{d_{(-)}^{+}}$of $\mathscr{N}\left(1-I_{\left.l_{-}^{+}\right)}(z, \kappa)\right)$ such that $\phi_{j_{(-)}}(\xi, \kappa)$ is analytic in $\xi$ for $0<\varepsilon<\varepsilon_{0}$ and for $j=1 \ldots d_{(-)}^{+}=\operatorname{dim} \mathscr{N}\left(1-I_{l_{(-)}^{+}}(z, \kappa)\right)$

$$
\phi_{\left.j_{(-)}\right)}\left(\varrho e^{i\left(\varphi_{(-)} \varepsilon\right)}, \kappa\right) \underset{\varepsilon \downarrow 0}{\longrightarrow} \phi_{j_{(-)}}(z, \kappa)
$$

in $H_{p_{D}}^{-s, 2} \otimes H_{k}^{s, 2}$ for $\varphi \neq 0$ and in $H^{-s, 2}$ for $\varphi=0$.

Proof. We consider the case of $I_{l+}$ for $\varphi<0$, the other cases are similar. Define the operators $B(\xi)$ and $K_{l}(\xi, \kappa)$ for $0<\varepsilon<\varepsilon_{0}$ by

$$
\begin{aligned}
B(\xi) & =\left(1+\xi^{2} p^{2}\right)^{2}\left(1-\xi^{-2} \Delta_{p_{D}}\right)^{-s / 2}\left(1-\xi^{-2} \Delta_{k_{D}}\right)^{s / 2}, \\
K_{l}(\xi, \kappa) & =B(\xi) I_{l}(\xi, \kappa) B^{-1}(\xi) .
\end{aligned}
$$


Then $K_{l}(\xi, \kappa)$ is analytic in $\xi$ for $0<\varepsilon<\varepsilon_{0}$ with values in $\mathscr{C}(\mathscr{H})$, and by Lemma 3.4

$$
K_{l}\left(\varrho e^{i(\varphi-\varepsilon)}, \kappa\right) \underset{\varepsilon \downarrow 0}{\longrightarrow} K_{l+}(z, \kappa)
$$

in the operator norm of $\mathscr{C}(\mathscr{H})$ where

$$
K_{l+}(z, \kappa)=B(z) I_{l+}(z, \kappa) B^{-1}(z) .
$$

Moreover for $\varrho>0$

$$
U(\varrho) K_{l}\left(e^{i(\varphi-\varepsilon)}, \kappa\right) U\left(\varrho^{-1}\right)=K_{l}\left(\varrho e^{i(\varphi-\varepsilon)}\right) .
$$

Evidently, $\sigma\left(K_{l}(\xi, \kappa)\right)=\sigma\left(I_{l}(\xi, \kappa)\right)$, and by analyticity and unitary equivalence for fixed $\varphi$, this spectrum is independent of $\xi$ for $0<\varepsilon<\varepsilon_{0}$. Hence we can choose a circle $\Gamma$ with center -1 and radius $\delta$ such that

and

$$
\{\zeta|0<| \zeta+1 \mid<\delta\} \cap \sigma\left(K_{l}(\xi, \kappa)\right)=\emptyset \text { for } 0<\varepsilon<\varepsilon_{0}
$$

$$
\{\zeta|0<| \zeta+1 \mid<\delta\} \cap \sigma\left(K_{l+}(z, \kappa)\right)=\emptyset .
$$

Define the projections $P(\xi, \kappa)$ and $P_{+}(z, \kappa)$ by

$$
P(\xi, \kappa)=-\frac{1}{2 \pi i} \int_{\Gamma}\left(K_{l}(\xi, \kappa)-\mu\right)^{-1} d \mu, P_{+}(z, \kappa)=-\frac{1}{2 \pi i} \int_{\Gamma}\left(K_{l+}(z, \kappa)-\mu\right)^{-1} d \mu .
$$

Then

$$
P\left(\varrho e^{i(\varphi-\varepsilon)}, \kappa\right) \underset{\varepsilon \downarrow 0}{\longrightarrow} P_{+}(z, \kappa)
$$

in the operator norm of $\mathscr{B}(\mathscr{H})$.

This implies that $\mathscr{N}\left(1-K_{l+}(z, \kappa)\right) \neq\{0\}$ if and only if

$$
\mathscr{N}\left(1-K_{l}(\xi, \kappa)\right) \neq\{0\} \text {. }
$$

Clearly

$$
\mathscr{N}\left(1-I_{l}(\xi, \kappa)\right)=B(\xi) \mathscr{N}\left(1-K_{l}(\xi, \kappa)\right)
$$

and

$$
\mathscr{N}\left(1-I_{l+}(z, \kappa)=B(\xi) \mathscr{N}\left(1-K_{l+}(z, \kappa) .\right.\right.
$$

On the other hand, it follows from Lemma 2.9 that $\mathscr{N}\left(1-I_{l}(\xi, \kappa)\right)$ is the same when $I_{l}(\xi, \kappa)$ is considered as an operator in $H_{p_{D}}^{-s, 2} \otimes H_{k_{D}}^{s, 2}$ and in $\mathscr{H}$, and hence

$$
\mathscr{N}\left(1-I_{l+}(z, \kappa) \neq\{0\} \quad \text { if and only if } \kappa \in \tilde{\mathscr{R}}_{\lambda}\right. \text {. }
$$

Let $\mathscr{E}$ be the dense set of dilation entire vectors. Since $P(\xi, \kappa)$ is finitedimensional, there exists for

$$
\psi=\psi\left(e^{i(\varphi-\varepsilon)}\right) \in \mathcal{N}\left(1-K_{l}\left(e^{i(\varphi-\varepsilon)}, \kappa\right)\right.
$$

a vector

$$
\chi=\chi\left(e^{i(\varphi-\varepsilon)} \in \mathscr{E}\right.
$$


such that

$$
\psi=P\left(e^{i(\varphi-\varepsilon)}, \kappa\right) \chi,
$$

and by unitary and analyticity

and

$$
\psi(\xi)=P(\xi, \kappa) \chi(\xi) \in \mathcal{N}\left(1-K_{l}(\xi, \kappa)\right)
$$

$$
\psi_{+}(z)=P_{+}(z, \kappa) \chi(z)=\lim _{\varepsilon \downarrow 0} \psi\left(\varrho e^{i(\varphi-\varepsilon)}\right) \in \mathcal{N}\left(1-K_{l+}(z, \kappa)\right) .
$$

To a basis $\left\{\psi_{i}\right\}$ of $\mathscr{N}\left(1-K_{l}\left(e^{i(\varphi-\varepsilon)}, \kappa\right)\right.$ corresponds bases $\psi_{i}(\xi)$ and $\psi_{i+}(z)$ of $\mathscr{N}\left(1-K_{l}(\xi, \kappa)\right)$ and $\mathscr{N}\left(1-K_{l+}(z, \kappa)\right)$ and hence bases

and

$$
\phi_{i}(\xi)=B(\xi) \psi_{i}(\xi)
$$

$$
\phi_{i+}(z)=B(z) \psi_{i+}(z)=\lim _{\varepsilon \downarrow 0} \phi_{i}\left(\varrho e^{i(\varphi-\varepsilon)}\right)
$$

of $\mathscr{N}\left(1-I_{l}(\xi, \kappa)\right)$ and $\mathscr{N}\left(1-I_{l+}(z, \kappa)\right)$, and the lemma is proved.

Lemma 3.9. For $\lambda=\lambda_{D}^{i}, \kappa=\lambda+\mu e^{2 i \varphi}, \mu \in \mathrm{R}^{+}$for $\varphi \neq 0$,

$$
\begin{gathered}
\kappa \in\left(\Sigma_{2}^{0}, E\right) \backslash \Sigma_{2} \text { for } \varphi=0, \\
\mathscr{N}\left(1-I_{\left.r_{(}+\right)}(z, \kappa)\right) \neq\{0\}
\end{gathered}
$$

if and only if

$$
\kappa \in \tilde{\mathscr{R}}_{\lambda} \cup \overline{\tilde{\mathscr{R}}}_{\lambda}^{\prime} \cup \tilde{\sigma}_{p}\left(\tilde{\mathscr{R}}_{\lambda}^{\prime} \cup \overline{\tilde{\mathscr{R}}}_{\lambda} \cup \tilde{\sigma}_{p}\right)
$$

where $I_{r \pm}(z, \kappa) \in \mathscr{C}\left(H^{s}\right)$.

Proof. This is proved in the same way as Lemma 3.8.

Lemma 3.10. For $\lambda=\lambda_{D}^{i}, \zeta=\lambda+\mu e^{2 i \varphi}, \mu \in \mathrm{R}^{+}, \zeta \notin \tilde{\mathscr{R}}_{\lambda} \cup \overline{\tilde{\mathscr{R}}}_{\lambda}^{\prime}\left(\tilde{\mathscr{R}}_{\lambda}^{\prime} \cup \overline{\tilde{\mathscr{R}}}_{\lambda}\right)$ for $\varphi \neq 0$ and $\zeta \in\left(\Sigma_{2}^{0}, E\right) \backslash\left(\Sigma_{2} \cup \tilde{\sigma}_{p}\right)$ for $\varphi=0$,

$$
\underset{(-)}{Y_{+}}(z, \zeta) \in \mathscr{B}\left(H^{s}, \tilde{H}^{s}\right)
$$

and for $\varphi \neq 0$

$$
Y_{\lambda_{(-)}^{+}}(z, \zeta) \in \mathscr{B}\left(H^{s}, \tilde{H}^{s}\right) \text {. }
$$

Proof. This follows from Lemma 3.6 and 3.9 and the fact that $X(z, \kappa) \in \mathscr{B}\left(H^{s}, \tilde{H}^{s}\right)$ (cf. the proof of Lemma 2.12).

Lemma 3.11. Under the conditions of Lemma 3.10

$$
\begin{array}{cl}
G_{+}(z, \zeta) Y_{(-)}(z, \zeta)=1 & \text { in } \mathscr{B}\left(H^{s}\right), \\
G_{\lambda_{(-)}^{+}}(z, \zeta) Y_{\lambda_{(-)}^{+}}(z, \zeta)=1 & \text { in } \mathscr{B}\left(H^{s}\right) .
\end{array}
$$

Proof. This follows from Lemmas 2.13, 2.15, 3.7, and 3.10.

We shall now establish the existence of limits $Y_{ \pm}\left(z, \lambda+\mu e^{2 i \varphi}\right)$ for $\varphi \neq 0$ in a different topology, related to the concept of smoothness. This will be utilized for the construction of a spectral measure of the operator $H(z)$. 
Lemma 3.12. For $\lambda=\lambda_{D}^{i}, \varphi \neq 0$ and $\Delta \subset \mathrm{R}^{+}$a bounded closed interval the following limits exist in $\mathscr{B}\left(\mathscr{H}, L^{2}\left(\Delta, H^{s}\right)\right)$, where $\mu \in \Delta$,

$$
\lim _{\varepsilon \downarrow 0} I_{r}\left(z, \lambda+\mu e^{2 i(\varphi \pm \varepsilon)}\right) .
$$

Proof. We consider the term given by (3.6) and (3.7). By repeated application of Lemma 2.1 and A1, A2 it is shown that

$$
V_{\alpha_{n-2}}(z) R_{D_{3}}\left(z, \lambda+\mu e^{2 i \varphi}\right) \cdot \ldots \cdot V_{\alpha_{1}}(z) R_{0}\left(z, \lambda+\mu e^{2 i \varphi}\right) \in \mathscr{B}\left(\mathscr{H}, H_{k_{D}}^{s} \otimes L_{p_{D}}^{2}\right)
$$

By a similar proof to that of Lemma 3.3

$$
V_{\alpha_{n-1}}(z) R_{D}^{E}(z, \zeta) \in \mathscr{B}\left(H_{k_{D}}^{s} \otimes L_{p_{D}}^{2}, H^{s}\right) .
$$

By a result of Kato [19] and Lavine [24], quoted in [8] Lemma 1.2, the following limits exist in $\mathscr{B}\left(L_{y_{D}}^{2}, L^{2}\left(\Delta, L_{y_{D}}^{2}\right)\right)$

$$
\lim _{\varepsilon \downarrow 0}\left(1+y_{D}^{2}\right)^{-s / 2}\left(-\Delta_{y_{D}}+\mu \pm i \varepsilon\right)^{-1} .
$$

From (3.12), Lemma 2.10(3) and A1, A2 follows the existence of the following limits in $\mathscr{B}\left(H_{k_{D}}^{s} \otimes L_{p_{D}}^{2}, L^{2}\left(\Delta, H^{s}\right)\right)$

$$
\lim _{\varepsilon \downarrow 0} V_{\alpha_{n-1}}(z)\left|\phi_{D}^{i}(z)\right\rangle r_{D}^{i}\left(z, \lambda+\mu e^{2 i(\varphi \pm \varepsilon)}\right)\left\langle\phi_{D}^{i}(\bar{z})\right| .
$$

The lemma follows from (3.10), (3.12), and (3.13).

Lemma 3.13. For $\lambda=\lambda_{D}^{i}, \varphi \neq 0$ and $\Delta \mathrm{CR}^{+}$a bounded, closed interval such that

$$
\left\{\lambda+e^{2 i \varphi} \Delta\right\} \cap\left\{\tilde{\mathscr{R}}_{\lambda} \cup \overline{\mathscr{R}}_{\lambda}^{\prime}\right\}\left(\left\{\tilde{\mathscr{R}}_{\lambda}^{\prime} \cup \overline{\mathscr{\mathscr { R }}}_{\lambda}\right\}\right)=\emptyset
$$

the following limits exist in $\mathscr{B}\left(\mathscr{H}, L^{2}\left(\Delta, H_{p_{D}}^{s}\right)\right)$,

$$
\lim _{\varepsilon \downarrow 0} Y_{D}^{i}\left(z, \lambda+\mu e^{2 i\left(\varphi_{(-\stackrel{+}{-} \varepsilon)}\right)}+\left\langle\phi_{D}^{i}(\bar{z})\right|\right\}
$$

and the following limits in $\mathscr{B}\left(\mathscr{H}, L^{2}(\Delta, \mathscr{H})\right)$

Proof. We write

$$
\lim _{\varepsilon \downarrow 0} Y_{\lambda}^{0}\left(z, \lambda+\mu e^{2 i\left(\varphi_{(-}^{+}{ }^{+} \varepsilon\right)}\right)
$$

$$
Y_{\lambda}(z, \zeta)=X_{\lambda}(z, \zeta)+X_{\lambda}(z, \zeta)\left(1-I_{r}(z, \zeta)\right)^{-1} I_{r}(z, \zeta)
$$

By Lemma 2.1 and A1, A2, $X_{\lambda}^{0}\left(z, \lambda+\mu e^{2 i \varphi}\right) \in \mathscr{B}(\mathscr{H})$.

Then the lemma follows from Lemmas 2.14, 3.6, 3.9, and 3.12 and (3.16).

We conclude this section by establishing the basic analycity properties of the operators $Y_{ \pm}(z, \zeta)$.

Lemma 3.14. For $\lambda=\lambda_{D}^{i} \in \Sigma_{2}, \lambda<E$, the $\mathscr{B}\left(H^{s}\right)$-valued functions $I_{\boldsymbol{r} \pm}\left(z, \lambda+\frac{z^{2}}{2 m_{D}}\right)$ are analytic in $\mathcal{O} \backslash \mathrm{R}^{+}$, and for $\lambda+\frac{\varrho^{2}}{2 m_{D}}<\lambda^{\prime}$

$$
\lim _{\varphi \rightarrow 0 \mp} I_{r \pm}\left(\varrho e^{i \varphi}, \lambda+\frac{\varrho^{2} e^{2 i \varphi}}{2 m_{D}}\right)=I_{r \pm}\left(\varrho, \lambda+\frac{\varrho^{2}}{2 m_{D}}\right) .
$$


Proof. We consider a typical term containing the singularity, obtained by setting $\zeta=\lambda+\frac{z^{2}}{2 m_{D}}$ in (3.6) and (3.7).

It is clear from Lemma 2.1 that for $D_{k} \in \mathscr{D}_{E^{\prime}}^{\prime}, R_{D_{k}}(z, \zeta)$ is analytic with values in $\mathscr{B}\left(H^{s}, H^{s, 2}\right)$ for $z \in \mathcal{O} \backslash\left(\left[2 m_{D}(E-\lambda)\right]^{1 / 2}, \infty\right)$.

For $D_{2} \in \mathscr{D}_{E}$ the operator $H_{D_{2}}(z)\left(1-P_{D_{2}}^{E}(z)\right)$ is analytic in $\mathcal{O}$, and

$$
\zeta \in \varrho\left(H_{D_{2}}(z)\left(1-P_{D_{2}}^{E}(z)\right) \mathscr{H}\right) \text {. }
$$

By the proof of Lemma 3.3 it is then easy to show that $R_{D_{2}}^{E}(z, \zeta)$ is analytic with values in $\mathscr{B}\left(H^{s}, H^{s, 2}\right)$ for $z \in \mathcal{O} \backslash\left(\left[2 m_{D}(E-\lambda)\right]^{1 / 2}, \infty\right)$.

By a straightforward proof, given in [8], Lemma 3.15, it is shown that for $\lambda_{D_{2}}^{j}<\lambda, r_{D_{2} \pm}^{j}(z, \zeta)$ is analytic with values in $\mathscr{B}\left(H_{p_{D_{2}}}^{s}, H_{p_{D_{2}}}^{-s, 2}\right)$ for $z \in \mathcal{O} \backslash \mathrm{R}^{+}$, and for $\varrho \in \mathrm{R}^{+}$

$$
\lim _{\varphi \rightarrow 0 \mp} r_{D_{2} \pm}^{j}\left(\varrho e^{i \varphi}, \lambda+\frac{\varrho^{2} e^{2 i \varphi}}{2 m_{D}}\right)=r_{D_{2} \pm}^{j}\left(\varrho, \lambda+\frac{\varrho^{2}}{2 m_{D}}\right) \text {; }
$$

for $\lambda_{D_{2}}^{j}=\lambda, r_{D_{2} \pm}^{j}(z, \zeta)$ is analytic for $z \in \mathcal{O}$, and for $\lambda_{D_{2}}^{j}>\lambda, r_{D_{2} \pm}^{j}(z, \zeta)$ is analytic for $z \in \mathcal{O} \backslash\left(\left[2 m_{D}\left(\lambda^{\prime}-\lambda\right)\right]^{1 / 2}, \infty\right)$.

From these observations and A1, A2 the lemma follows.

Definition 3.14. For $\lambda=\lambda_{D}^{i} \in \Sigma_{2}$ and any set $D(\lambda) \subset \mathrm{C}$, we use the notation

$$
D(\lambda)^{2}=\left\{z \in \mathcal{O} \mid \lambda+\frac{z^{2}}{2 m_{D}} \in D(\lambda)\right\} .
$$

Lemma 3.15. For $\lambda \in \Sigma_{2}, \lambda<E$, the $\mathscr{B}\left(H^{s}, \tilde{H}^{s}\right)$-valued function

$$
Y_{+(-)}\left(z, \lambda+\frac{z^{2}}{2 m_{D}}\right)
$$

is meromorphic in $\mathcal{O} \backslash \mathrm{R}^{+}$with poles at most at points of

$$
\left(\tilde{\mathscr{R}}_{\lambda} \cup \overline{\tilde{\mathscr{R}}}_{\lambda}^{\prime}\right)^{\ulcorner}\left(\left(\tilde{\mathscr{R}}_{\lambda}^{\prime} \cup \overline{\tilde{\mathscr{R}}}_{\lambda}\right)^{\nu}\right) \text {. }
$$

Moreover, for $\lambda+\frac{\varrho^{2}}{2 m_{D}} \in\left(\lambda, \lambda^{\prime}\right) \mid \tilde{\sigma}_{p}$

$$
\lim _{\varphi \rightarrow 0 \mp} Y_{ \pm}\left(\varrho e^{i \varphi}, \lambda+\frac{\varrho^{2} e^{2 i \varphi}}{2 m_{D}}\right)=Y_{ \pm}\left(\varrho, \lambda+\frac{\varrho^{2}}{2 m_{D}}\right) .
$$

Proof. This follows from Lemmas 2.10(2), 3.9, 3.10, and 3.13.

4. Isomorphism of $\mathscr{N}\left(1+R_{D_{+}}(z, \zeta) V_{\dot{D}}(z)\right)$

and $\mathscr{N}\left(G_{+}(z, \zeta) / \mathcal{N}\left(J(z) R_{1_{+}}(z, \zeta)\right)\right.$

Definition 4.1. $\Sigma_{D}=\left\{p_{D} \in \mathrm{R}_{p_{D}}^{m}|| \mathrm{p}_{D} \mid=1\right\}$,

$$
h_{D}^{i}=h_{D}=L^{2}\left(\Sigma_{D}\right) \quad \text { for } \quad i=1 \ldots n(D) \text {. }
$$


For $\lambda \in \Sigma_{2}, \lambda<E, \mu \in\left[\lambda, \lambda^{\prime}\right)$, let

$$
\begin{aligned}
& h(\mu)=\sum_{D \in \mathscr{D} E} \sum_{\lambda_{D}^{i} \leqq \lambda} \oplus h_{D}^{i}, \\
& T_{D}(\mu)=\left(2 m_{D}\right)^{m / 4} 2^{-1 / 2} \mu^{\frac{m-2}{4}} \gamma_{D}\left(\left(2 m_{D} \mu\right)^{1 / 2}\right) \in \mathscr{B}\left(H_{p_{D}}^{s}, h_{D}\right) \text { for } \mu \in R^{+}, \\
& \quad\left(T_{D}^{i} f\right)(\mu)=T_{D}\left(\mu-\lambda_{D}^{i}\right) f \text { for } f \in H_{P_{D}}^{s}, \quad \mu \in\left(\lambda_{D}^{i}, \infty\right) .
\end{aligned}
$$

For $\mu \in\left(\lambda, \lambda^{\prime}\right)$ set

$$
\begin{gathered}
T(\mu)=\sum_{D \in \mathscr{D}_{E}} \sum_{\lambda_{D}^{i} \leqq \lambda} \oplus T_{D}^{i}(\mu), \\
T=\sum_{D \in \mathscr{D}_{E}} \sum_{i=1}^{n(D)} \oplus T_{D}^{i} .
\end{gathered}
$$

The operators $T_{D}^{i}$ are defined such that $T_{D}^{i}$ has a unitary extension in $\mathscr{B}\left(L_{p_{D}}^{2}, L^{2}\left(\left(\lambda_{D}^{i}, \infty\right), h_{D}^{i}\right)\right.$, and such that

$$
T H_{1} T^{-1}=\lambda \quad \text { in } \quad L^{2}\left(\left(\Sigma_{2}^{0}, \infty\right), h(\lambda)\right) .
$$

By (1.15), trace and dilation operators are connected by

$$
T_{D}(\mu)=2^{-1 / 2} \mu^{-1 / 2} \gamma_{D}(1) U_{D}\left(\left(2 m_{C_{1}, C_{2}} \mu\right)^{1 / 2}\right),
$$

where $\left\{U_{D}(\varrho)\right\}_{\varrho \in \mathrm{R}^{+}}$is the dilation group in $\mathscr{H}_{p_{D}}$.

Lemma 4.2. For $z \in \mathcal{O}, \zeta=\lambda_{D}^{i}+\mu e^{2 i \varphi}$,

$$
e^{2 i \varphi}(2 \pi i)^{-1}\left\{r_{D+}^{i}(z, \zeta)-r_{D-}^{i}(z, \zeta)\right\}=T_{D}^{*}(\mu) T_{D}(\mu) .
$$

Proof. This follows from a well-known formula of scattering theory, see [23], Proposition 5.5.1.

Lemma 4.3. Let $\lambda=\lambda_{D}^{i} \in \Sigma_{2}, \lambda<E$. For $\varphi=0$, let $\varrho \in \mathrm{R}^{+}, \mu \in\left(0, \lambda^{\prime}-\lambda\right), \tilde{u} \in \tilde{H}^{s}$ and assume

$J(\varrho) R_{1_{(-)},}(\varrho, \lambda+\mu) \tilde{u} \in \mathscr{H}$.

Then for $\lambda_{D^{\prime}}^{j} \leqq \lambda$

$$
\begin{gathered}
\gamma_{D}\left(\varrho^{-1}\left(2 m_{D}\left(\lambda-\lambda_{D^{\prime}}^{j}+\mu\right)\right)^{1 / 2}\right) \tau_{D^{\prime}}^{j}=0, \\
\text { in particular for } \varrho=1, T(\lambda+\tilde{\mu}) u=0 .
\end{gathered}
$$

For $\varphi \neq 0$, let $\varrho \in R^{+}, \mu \in \mathrm{R}^{+}$and assume

$$
J_{\lambda}(z) R_{1 \lambda_{(-)}^{+}}\left(z, \lambda+\mu e^{2 i \varphi}\right) \tilde{u} \in \mathscr{H} .
$$

Then $T_{D}\left(\mu \varrho^{-2}\right) \tau=0$,

$$
\text { in particular for } \varrho=\left(2 m_{D} \mu\right)^{1 / 2}, \gamma_{D}(1) \tau=0 \text {. }
$$

Proof. This is proved in the same way as [8], Lemma 5.4.

Lemma 4.4. Let $\tilde{u} \in \tilde{H}^{s}$ for $\varphi=0, \tilde{u} \in \tilde{H}_{\lambda}^{s}$ for $\varphi \neq 0$. For $\varphi=0, \lambda \in \Sigma_{2}, \quad \lambda<E$, $\mu \in\left(\lambda, \lambda^{\prime}\right) \backslash \tilde{\sigma}_{p}$,

$$
T(\mu) Y_{ \pm}(\mu) G_{ \pm}(\mu) \tilde{u}=T(\mu) \tilde{u}
$$


For $\varphi \neq 0, \zeta=\lambda+\mu e^{2 i \varphi}, \zeta \notin \tilde{\mathscr{R}}_{\lambda} \cup \overline{\mathscr{R}}_{\lambda}^{\prime}\left(\tilde{\mathscr{R}}_{\lambda}^{\prime} \cup \overline{\mathscr{R}}_{\lambda}\right)$,

$$
T_{D}\left(\mu \varrho^{-2}\right) Y_{\lambda_{( \pm)}}(z, \zeta) G_{\lambda_{( \pm)}}(z, \zeta) \tilde{u}=T_{D}\left(\mu \varrho^{-2}\right) \tau,
$$

in particular for $\varrho=\left(2 m_{D} \mu\right)^{1 / 2}$

$$
\gamma_{D}(1) Y_{\lambda_{(-)}^{+}}\left(z, \lambda+\frac{z^{2}}{2 m_{D}}\right) \underset{(-)}{G_{(-)}}\left(z, \lambda+\frac{z^{2}}{2 m_{D}}\right) \tilde{u}=\gamma_{D}(1) \tau .
$$

Proof. By Lemmas 2.12-2.15, 3.7, and 3.10

$$
J R_{1 \pm}(\mu) Y_{ \pm}(\mu) G_{ \pm}(\mu) \tilde{u}=J R_{1 \pm}(\mu) \tilde{u}
$$

and for $\varphi \neq 0$

$$
J_{\lambda}(z) R_{1 \lambda \pm}(z, \zeta) Y_{\lambda \pm}(z, \zeta) G_{\lambda \pm}(z, \zeta) \tilde{u}=J_{\lambda}(z) R_{1 \lambda \pm}(z, \zeta) \tilde{u},
$$

and the lemma follows from Lemma 4.3.

Definition 4.5. For $\lambda \in \Sigma_{2}, \lambda<E, \zeta=\lambda+\mu e^{2 i \varphi}$,

$$
\tilde{\mathcal{N}}\left(G_{ \pm}(z, \zeta)\right)=\mathscr{N}\left(G_{ \pm}(z, \zeta)\right) / \mathcal{N}\left(J(z) R_{1 \pm}(z, \zeta)\right) .
$$

For $\varphi=0$,

$$
\mathscr{N}_{0}\left(G_{ \pm}(\mu)\right)=\left\{\Omega \in \mathscr{N}\left(G_{ \pm}(\mu) \mid T(\mu) \Omega=0\right\} .\right.
$$

For $\varphi \neq 0$,

$$
\mathscr{N}_{0}\left(G_{\lambda \pm}(z, \zeta)\right)=\left\{\Omega \in \mathscr{N}\left(G_{\lambda \pm}(z, \zeta)\right) \mid T_{D}\left(\mu \varrho^{-2}\right) \tau=0\right\}
$$

Moreover, in accordance with Lemma 4.3 we set

$$
\tilde{\mathcal{N}}_{0}\left(G_{ \pm}(z, \zeta)\right)=\mathscr{N}_{0}\left(G_{ \pm}(z, \zeta)\right) / \mathscr{N}\left(J(z) R_{1 \pm}(z, \zeta)\right) \text {. }
$$

The spaces $\tilde{\mathcal{N}}\left(G_{\lambda \pm}(z, \zeta)\right)$ and $\tilde{\mathscr{N}}_{0}\left(G_{\lambda \pm}(z, \zeta)\right)$ are defined similarly.

Lemma 4.6. Let $\varphi \neq 0, \lambda=\lambda_{D}^{i}, \kappa=\lambda+\mu e^{2 i \varphi}$, and consider $R_{D+}(z, \kappa) V_{D}^{\prime}(z)$ in accordance with Lemmas 3.2, 3.3 as an operator in $\mathscr{B}\left(H_{p_{D}}^{-s, 2} \otimes H_{k_{D}}^{s, 2}\right)$.

The operator $J_{\lambda}(z) R_{1, \lambda+}(z, \kappa)$ is an isomorphism of $\tilde{\mathcal{N}}\left(G_{\lambda+}(z, \kappa)\right)$ onto $\mathscr{N}\left(1+R_{D+}(z, \kappa) V_{D}^{\prime}(z)\right)$ with the inverse $M_{\kappa}$ given by

$$
M_{\kappa} \Psi=\Omega=\{u, \tau\}
$$

where

$$
u=-\left(1-P_{D}^{i}(z)\right) V_{D}^{\prime}(z) \Psi+V_{D}(z) R_{D}^{\lambda}(z, \kappa) V_{D}^{\prime}(z) \Psi
$$

and

$$
\tau=-\left\langle\phi_{D}^{i}(\bar{z})\right| V_{D}^{\prime}(z) \Psi
$$

Furthermore,

$$
\tilde{\mathscr{N}}_{0}\left(G_{\lambda+}(z, \kappa)\right)=\tilde{\mathscr{N}}_{0}\left(G_{\lambda_{-}}(z, \kappa)\right)
$$


Assume moreover, that A1, A2 hold with $W_{\alpha}(z) \in \mathscr{B}\left(H^{s^{\prime}, 2}, H^{s^{\prime}}\right)$ for $0 \leqq s^{\prime} \leqq 1 / 2$. Then $J(z) R_{1, \lambda+}(z, \kappa)$ maps $\tilde{\mathscr{N}}_{0}\left(G_{\lambda+}(z, \kappa)\right)$ isomorphically onto $\mathscr{N}(H(z)-\kappa)$. The same holds with + replaced by - , and

$$
\mathscr{N}\left(1+R_{D+}(z, \kappa) V_{D}^{\prime}(z)\right) \cap \mathcal{N}\left(1+R_{D-}(z, \kappa) V_{D}^{\prime}(z)\right)=\mathscr{N}(H(z)-\kappa) .
$$

Proof. It follows from Lemmas 3.1 and 3.3 that

$$
M_{\kappa} \in \mathscr{B}\left(H_{p_{D}}^{-s, 2} \oplus H_{k_{D}}^{s, 2}, \tilde{H}_{\lambda}^{s}\right)
$$

and by [8], Lemma 1.1

$$
J_{\lambda}(z) R_{1, \lambda+}(z, \kappa) \in \mathscr{B}\left(H_{\lambda}^{s}, H_{p_{D}}^{-s, 2} \otimes H_{k_{D}}^{s, 2}\right) .
$$

For $\Omega \in \mathscr{N}\left(G_{\lambda_{+}}(z, \kappa)\right)$ we have by $(2.35)$

$$
\begin{aligned}
\left(1+R_{D+}(z, \kappa) V_{D}^{\prime}(z)\right) J_{\lambda}(z) R_{1, \lambda+}(z, \kappa) \Omega & =R_{D+}(z, \kappa) G_{\lambda+}(z, \kappa) \Omega \\
& =0 \text { in } H_{p_{D}}^{-s, 2} \otimes H_{k_{D}}^{s, 2} .
\end{aligned}
$$

On the other hand, let $\Psi \in H_{p_{D}}^{-s, 2} \otimes H_{k_{D}}^{s, 2}$ and

$$
\left(1+R_{D+}(z, \kappa) V_{D}^{\prime}(z)\right) \Psi=0 .
$$

By definition of $M_{\kappa},(4.15)$ can be written

$$
\Psi=J_{\lambda}(z) R_{1, \lambda+}(z, \kappa) M_{\kappa} \Psi .
$$

By (4.15) and (4.16)

$$
\begin{aligned}
G_{\lambda+}(z, \kappa) M_{\kappa} \Psi= & \lim _{\varepsilon \downarrow 0}\left(H\left(\varrho e^{i(\varphi-\varepsilon)}-\kappa\right) \Psi\right. \\
= & -\lim _{\varepsilon \downarrow 0}\left(H_{D}\left(\varrho e^{i(\varphi-\varepsilon)}\right)-\kappa\right) R_{D+}(z, \kappa) V_{D}^{\prime}(z) \Psi \\
& +\lim _{\varepsilon \downarrow 0} V_{D}^{\prime}\left(\varrho e^{i(\varphi-\varepsilon)}\right) \Psi=0,
\end{aligned}
$$

and the isomorphism of $\mathscr{N}\left(1+R_{D+}(z, \kappa) V_{D}^{\prime}(z)\right)$ and $\tilde{\mathscr{N}}\left(G_{+}(z, \kappa)\right)$ is proved.

If $\Omega \in \tilde{\mathscr{N}}_{0}\left(G_{\lambda+}(z, \kappa)\right)$, then by Lemma 4.2

$$
\Psi=J(z) R_{1, \lambda+}(z, \kappa) \Omega=J(z) R_{1, \lambda-}(z, \kappa) \Omega
$$

and hence

$$
M_{\kappa} \Psi=\tilde{\Omega} \in \tilde{N}_{0}\left(G_{\lambda-}(z, \kappa)\right) .
$$

If $\Psi \in \mathscr{N}(H(z)-\kappa)$, i.e. $\Psi \in \mathscr{N}\left(1+R_{D+}(z, \kappa)\right)$ and $\Psi \in H^{0,2}$, and $\Omega=M_{\kappa} \Psi$, it follows from Lemma 4.3 and (4.16) that $T_{D}\left(\mu \varrho^{-2}\right) \tau=0$, hence $\Omega \in \tilde{\mathscr{N}}_{0}\left(G_{\lambda+}(z, \kappa)\right)$.

Suppose on the other hand, that $\Omega \in \tilde{\mathscr{N}}_{0}\left(G_{\lambda+}(z, \kappa)\right)$, and let

$$
\Psi=J_{\lambda}(z) R_{1, \lambda+}(z, \kappa) \Omega .
$$

By a result of Agmon [1], quoted as [8], Lemma 1.5, $\Psi \in H_{p_{D}}^{s-1,2} \otimes H_{k_{D}}^{s, 2}$. Then by Lemma 3.1 extended on the basis of A1, A2 in the strengthened form, $\tau \in H_{p_{D}}^{3 s-1}$. Repeating this argument, we get after $k$ steps

$$
\Psi \in H_{p_{D}}^{s-1+k(2 s-1), 2} \otimes H_{k_{D}}^{s, 2},
$$


hence $\Psi \in H^{0,2}$, and we have proved that $J_{\lambda}(z) R_{1, \lambda+}(z, \kappa)$ is an isomorphism of $\tilde{N}_{0}\left(G_{\lambda+}(z, \kappa)\right)$ onto $\mathscr{N}(H(z)-\kappa)$. The same proof holds with + replaced by - , where $M_{\kappa}$ is defined by (4.12)-(4.14).

It is clear that

$$
\mathscr{N}(H(z)-\kappa) \subseteq \mathscr{N}\left(1+R_{D \pm}(z, \kappa) V_{D}^{\prime}(z)\right) .
$$

Conversely, if $\left.\Psi \in \mathscr{N}\left(1+R_{D \pm}(z, \kappa)\right) V_{D}^{\prime}(z)\right)$, and $M_{\kappa} \Psi=\Omega$, then by (4.16)

$$
\Psi=J_{\lambda}(z) R_{1, \lambda+}(z, \kappa) \Omega=J_{\lambda}(z) R_{1, \lambda-}(z, \kappa) \Omega .
$$

This implies

$$
\left|\phi_{D}^{i}(z)\right\rangle\left(r_{D+}^{i}(z, \kappa)-r_{D-}^{i}(z, \kappa)\right) \tau=0
$$

and hence $T_{D}\left(\mu \varrho^{-2}\right) \tau=0$.

As seen above, this implies $\Psi \in \mathscr{N}(H(z)-\kappa)$, and the lemma is proved.

Lemma 4.7. Let $\varphi \neq 0, \lambda=\lambda_{D}^{i} \in \Sigma_{2}, \kappa=\lambda+\mu e^{2 i \varphi}$, and consider $R_{D+}(z, \kappa) V_{D}^{\prime}(z)$ as an operator in $\mathscr{B}\left(H_{p_{D}}^{-s, 2} \otimes H_{k_{D}}^{s, 2}\right)$.

There exist $\varepsilon_{0}>0$ and for $0<\varepsilon<\varepsilon_{0}$ bases $\left\{\Psi_{i}(\xi)\right\}$ of $\mathscr{N}(H(\xi)-\kappa), \xi=\varrho e^{i(\varphi-\varepsilon)}$, and a basis $\left\{\Psi_{i+}(z)\right\}$ of $\mathcal{N}\left(1+R_{D+}(z, \kappa) V_{D}^{\prime}(z)\right)$, such that $\Psi_{i}(\xi)$ is analytic for $\varrho>0$, $0<\varepsilon<\varepsilon_{0}$, and

$$
\lim _{\varepsilon \downarrow 0} \Psi_{i}\left(\varrho e^{i(\varphi-\varepsilon)}\right)=\Psi_{i+}(z) \quad \text { in } \quad H_{p_{D}}^{-s, 2} \otimes H_{k_{D}}^{s, 2} .
$$

The same holds with + replaced by - and $\varphi-\varepsilon$ by $\varphi+\varepsilon$.

If $\kappa \in \sigma_{p}(H(z))$, there exist $\varepsilon_{1}, \varepsilon_{2}$ and functions $\Psi_{i}(\xi), \xi=\varrho e^{i \alpha}$, analytic with values in $H_{p_{D}}^{-s, 2} \otimes H_{k_{D}}^{2, s}$ for $\varrho \in \mathrm{R}^{+}, \varphi-\varepsilon_{1}<\alpha<\varphi+\varepsilon_{2}$, and such that $\left\{\Psi_{i}(z)\right\}$ is a basis of $\mathscr{N}(H(z)-\kappa)$, and $\left\{\Psi_{i}(\xi)\right\}$ generates a subspace of $\mathscr{N}(H(\xi)-\kappa)$ for $\varphi-\varepsilon_{1}<\alpha<\varphi$ and $\varphi<\alpha<\varphi+\varepsilon_{2}$.

Proof. Since

$$
1-I_{l}(\xi, \kappa)=D(\xi, \kappa)(H(\xi)-\kappa)
$$

we have

$$
\mathscr{N}\left(1+R_{D}(\xi, \kappa) V_{D}^{\prime}(\xi)\right)=\mathscr{N}(H(\xi)-\kappa) \subseteq \mathscr{N}\left(1-I_{l}(\xi, \kappa)\right) .
$$

Moreover,

$$
\mathscr{N}\left(1+R_{D+}(z, \kappa) V_{D}^{\prime}(z)\right) \subseteq \mathscr{N}\left(1-I_{l+}(z, \kappa)\right),
$$

which can be seen as follows.

Let

$$
\Psi+R_{D+}(z, \kappa) V_{D}^{\prime}(z) \Psi=0, \quad \Psi \in H_{p_{D}}^{-s, 2} \otimes H_{k_{D}}^{s, 2} .
$$

Then by Lemma 3.1, $V_{D}^{\prime}(z) \Psi \in H^{s}$, and

$$
\begin{aligned}
\lim _{\varepsilon \downarrow 0}\left(H\left(\varrho e^{i(\varphi-\varepsilon)}\right)-\kappa\right)= & -\lim _{\varepsilon \downarrow 0}\left(H_{D}\left(\varrho e^{i(\varphi-\varepsilon)}\right)-\kappa\right) R_{D+}(z, \kappa) V_{D}^{\prime}(z) \Psi \\
& +\lim _{\varepsilon \downarrow 0} V_{D}^{\prime}\left(\varrho e^{i(\varphi-\varepsilon)}\right) \Psi \\
= & -V_{D}^{\prime}(z) \Psi+V_{D}^{\prime}(z) \Psi=0 \text { in } H^{s} .
\end{aligned}
$$


Hence by Lemmas 2.1, 3.2, and 3.3

$$
\begin{aligned}
\lim _{\varepsilon \downarrow 0}\left(1-I_{l}\left(\varrho e^{i(\varphi-\varepsilon)}, \kappa\right)\right) \Psi & =\lim _{\varepsilon \downarrow 0} D\left(\varrho e^{i(\varphi-\varepsilon)}, \kappa\right)\left(H\left(\varrho e^{i(\varphi-\varepsilon)}-\kappa\right) \Psi\right. \\
& =0 \text { in } \quad H_{p_{D}}^{-s, 2} \otimes H_{k_{D}}^{s, 2} .
\end{aligned}
$$

For a fixed $\varepsilon_{1} \in\left(0, \varepsilon_{0}\right)$, let $\left\{\Psi_{i}\left(e^{i\left(\varphi-\varepsilon_{1}\right)}\right)\right\}$ be a basis of $\mathcal{N}\left(H\left(e^{i\left(\varphi-\varepsilon_{1}\right)}\right)-\kappa\right)$. Then by (4.17) and Lemma 3.8 there exist analytic functions $\Psi_{i}(\xi)$ and limiting functions $\Psi_{i+}(z)$ such that

and

$$
\Psi_{i}\left(\varrho e^{i(\varphi-\varepsilon)}\right) \underset{\varepsilon \downarrow 0}{\longrightarrow} \Psi_{i+}(z) \quad \text { in } \quad H_{p_{D}}^{-s, 2} \otimes H_{k_{D}}^{s, 2}
$$

$$
\Psi_{i}\left(\varrho e^{i\left(\varphi-\varepsilon_{1}\right)}\right)=U(\varrho) \Psi_{i}\left(e^{i\left(\varphi-\varepsilon_{1}\right)}\right) .
$$

By analyticity of $R_{D}(\xi, \kappa) V_{D}^{\prime}(\xi)$ this implies

$$
\left(1+R_{D}(\xi, \kappa) V_{D}^{\prime}(\xi)\right) \Psi_{i}(\xi)=0
$$

and then for $\varepsilon \downarrow 0$

$$
\left(1+R_{D+}(z, \kappa) V_{D}^{\prime}(z)\right) \Psi_{i+}(z)=0 .
$$

It is clear, that $\left\{\Psi_{i}(\xi)\right\}$ is a basis of $\mathscr{N}(H(\xi)-\kappa)$ for all $\xi$ with $0<\varepsilon<\varepsilon_{0}$ (we could start from any $\left.\varepsilon \in\left(0, \varepsilon_{0}\right)\right)$, and that $\left\{\Psi_{i+}(z)\right\}$ is a set of linearly independent vectors. Conversely, a basis $\left\{\tilde{\Psi}_{i}(z)\right\}$ of $\mathscr{N}\left(1+R_{D^{+}}(z, \kappa) V_{D}^{\prime}(z)\right)$ by (4.18) and Lemma 3.8 is boundary value of a linearly independent set of analytic vectors $\left\{\tilde{\Psi}_{i}(\xi)\right\}$, which by analytic continuation are in $\mathscr{N}\left(1+R_{D}(\xi, \kappa) V_{D}^{\prime}(\xi)\right)$. Thus $\left\{\Psi_{i}(z)\right\}$ is a basis of $\left.\mathscr{N}\left(1+R_{D+}(z, \kappa)\right) V_{D}^{\prime}(z)\right)$.

The same proof holds with + replaced by - and $\varphi-\varepsilon$ by $\varphi+\varepsilon$.

If $\kappa \in \sigma_{p}(H(z))$, by Lemma 4.6

$$
\mathscr{N}(H(z)-\kappa) \leqq \mathscr{N}\left(1+R_{D+}(z, \kappa) V_{D}^{\prime}(z)\right) \cap \mathscr{N}\left(1+R_{D-}(z, \kappa) V_{D}^{\prime}(z)\right) .
$$

Then the above construction yields a basis $\left\{\Psi_{i}(z)\right\}$ of $\mathscr{N}(H(z)-\kappa)$ and functions $\Psi_{i}^{(1)}(\xi), \xi=\varrho e^{i \alpha}$ analytic for $\varphi-\varepsilon_{1}<\alpha<\varphi$ and $\Psi_{i}^{(2)}(\xi)$, analytic for $\varphi<\alpha$ $<\varphi+\varepsilon_{2}$, such that

$$
\begin{aligned}
& \lim _{\varepsilon \downarrow 0} \Psi_{i}^{(1)}\left(\varrho e^{i(\varphi-\varepsilon)}\right)=\Psi_{i}(z) \text { in } \quad H_{p_{D}}^{-s, 2} \otimes H_{k_{D}}^{s, 2} \\
& \lim _{\varepsilon \downarrow 0} \Psi_{i}^{(2)}\left(\varrho \mathrm{e}^{i(\varphi+\varepsilon)}\right)=\Psi_{i}(z) \text { in } \quad H_{p_{D}}^{-s, 2} \otimes H_{k_{D}}^{s, 2}
\end{aligned}
$$

It follows, that $\Psi_{i}(\xi)$ defined by

$$
\begin{array}{rll}
\Psi_{i}^{(1)}\left(\varrho e^{i \alpha}\right) & \text { for } & \varphi-\varepsilon_{1}<\alpha<\varphi \\
\left.\Psi_{i}(\xi)=\right) \Psi_{i}\left(\varrho e^{i \alpha}\right) & \text { for } & \alpha=\varphi \\
\Psi_{i}^{(2)}\left(\varrho e^{i \alpha}\right) & \text { for } & \varphi<\alpha<\varphi+\varepsilon_{2}
\end{array}
$$

is analytic in $\xi$, and the lemma is proved.

Lemma 4.8. For $\mu \in\left(\Sigma_{2}^{0}, E\right) \backslash \Sigma_{2}$,

$$
\begin{aligned}
& \tilde{\mathcal{N}}\left(G_{+}(\mu)\right)=\tilde{\mathscr{N}}_{0}\left(G_{+}(\mu)\right)=\tilde{\mathscr{N}}_{0}\left(G_{-}(\mu)\right)=\tilde{\mathcal{N}}\left(G_{-}(\mu)\right), \\
& J R_{1_{+}}(\mu) \tilde{u}=J R_{1_{-}}(\mu) \tilde{u} \quad \text { for } \quad \tilde{u} \in \tilde{\mathcal{N}}\left(G_{ \pm}(\mu)\right),
\end{aligned}
$$


and $J R_{1 \pm}(\mu)$ is an isomorphism of $\tilde{N}\left(G_{ \pm}(\mu)\right)$ onto $\mathcal{N}(H-\mu)$ with the inverse $M_{\mu}$ given by

$$
M_{\mu} \Psi=\tilde{\Omega}, \text { where } \Omega=\{u, 0, \ldots, 0\}, \quad u=-V \Psi .
$$

Proof. It follows from Lemmas 2.9, 2.10, and 3.8, that $\mathscr{N}(H-\mu)$ coincides with $\mathscr{N}\left(1+R_{0}(\mu) V\right)$ where $1+R_{0}(\mu) V$ is considered as an operator in $H^{-s, 2}$, and $\mathscr{N}(H-\mu) \subseteq H^{s, 2}$.

If $\Omega \in \tilde{\mathcal{N}}\left(G_{+}(\mu)\right)$, then $J R_{1+}(\mu) \Omega \in H^{-s, 2}$, and

$$
\left(1+R_{0}(\mu) V\right) R_{1+} \Omega=R_{0}(\mu) G_{+}(\mu) \Omega=0,
$$

hence

$$
\Psi=J R_{1+}(\mu) \Omega \in \mathscr{N}\left(1+R_{0}(\mu) V\right)=\mathscr{N}(H-\mu) .
$$

By Lemma 4.3 this implies $T(\mu) \Omega=0$, so $\tilde{\Omega} \in \tilde{\mathscr{N}}_{0}\left(G_{+}(\mu)\right)$.

Clearly, $\tilde{\mathcal{N}}_{0}\left(G_{+}(\mu)\right)=\tilde{\mathscr{N}}_{0}\left(G_{-}(\mu)\right)$, since

$$
G_{+}(\mu)-G_{-}(\mu)=W\left\{R_{1+}(\mu)-R_{1-}(\mu)\right\},
$$

and we have shown (4.20) and hence (4.21).

If $\Psi \in \mathscr{N}(H-\mu)$, then $\Psi \in H^{s, 2}$, and $-V \Psi \in H^{s}$, so $\Omega \in \tilde{H}^{s}, \Psi=J R_{1 \pm}(\mu) \Omega$, and

$$
G_{ \pm}(\mu) \Omega=(H-\mu) J R_{1 \pm}(\mu) \Omega=(H-\mu) \Psi=0,
$$

and the lemma is proved.

\section{Construction of Wave Operators and $S$-Matrix}

Lemma 5.1. Let $\lambda=\lambda_{D}^{i}$, and let $\Delta$ be a Borel set such that $\bar{\Delta}$ is a compact subset of $\mathrm{R}^{+}$ for $\varphi \neq 0$ and of $\left(0, \lambda^{\prime}-\lambda\right)$ for $\varphi=0$, and such that

$$
\left(\lambda+\Delta e^{2 i \varphi}\right) \cap\left(\tilde{\mathscr{R}}_{\lambda} \cup \tilde{\mathscr{R}}_{\lambda}^{\prime} \cup \tilde{\sigma}_{p}(H) \cup \overline{\mathscr{R}}_{\lambda} \cup \overline{\tilde{\mathscr{R}}}_{\lambda}^{\prime}\right)=\emptyset .
$$

Then for $f, g \in \mathscr{H}$ and for $f$ and/or $g$ replaced by functions in $L^{2}\left(\Delta, H^{s}\right)$ we have for $\varphi \neq 0$

$$
\begin{aligned}
& e^{-2 i \varphi} \int_{\Delta}\left(T_{D}(\mu) Y_{D \pm}^{i}\left(\varphi, \lambda+\mu e^{2 i \varphi}\right) f, T_{D}(\mu) Y_{D \pm}^{i}\left(-\varphi, \lambda+\mu e^{-2 i \varphi}\right) g\right)_{h_{D}} d \mu \\
& =\lim _{\varepsilon \downarrow 0} \frac{1}{2 \pi i} \int_{\Delta}\left(\left\{R\left(\varphi, \lambda+(\mu+i \varepsilon) e^{2 i \varphi}\right)-R\left(\varphi, \lambda+(\mu-i \varepsilon) e^{2 i \varphi}\right)\right\} f, g\right) d \mu .
\end{aligned}
$$

$\operatorname{For} \varphi=0$

$$
\begin{gathered}
\sum_{D \in \mathscr{D}_{E}} \sum_{\lambda{ }_{D} \leqq \lambda} \int_{\Delta}\left(T_{D}^{i}(\lambda+\mu) Y_{D \pm}^{i}(\lambda+\mu) f, T_{D}^{i}(\lambda+\mu) Y_{D \pm}^{i}(\lambda+\mu) g\right)_{h_{D}} d \mu \\
=\lim _{\varepsilon \downarrow 0} \frac{1}{2 \pi i} \int_{\Delta}(\{R(\lambda+\mu+i \varepsilon)-R(\lambda+\mu-i \varepsilon)\} f, g) d \mu .
\end{gathered}
$$

The left hand side of (5.1) or (5.2) defines a bounded, sesquilinear form on $\mathscr{H} \times \mathscr{H}$. 
Proof. This is proved in the same way as [8], Lemma 7.1, utilizing Lemmas 2.12, $2.14,3.10,3.13$, and 4.2 , and writing $\chi_{\Delta}\left\langle\phi_{D}^{i}(\bar{z})\right| f$ as a function in $L^{2}\left(\Delta, h_{D}\right)$.

Definition 5.2. For $\varphi \neq 0$ and $\lambda \in \Sigma_{2}, \lambda<E$, we denote by $\mathscr{G}_{\lambda}(\varphi)$ the set of all Borel sets $\Delta$ such that $\bar{\Delta}$ is a compact subset of $\mathrm{R}^{+}$and

$$
\left(\lambda+e^{2 i \varphi} \bar{\Delta}\right) \cap\left(\tilde{\mathscr{R}}_{\lambda} \cup \tilde{\mathscr{R}}_{\lambda}^{\prime} \cup \overline{\tilde{\mathscr{R}}}_{\lambda} \cup \overline{\tilde{\mathscr{R}}}_{\lambda}^{\prime}\right)=\emptyset .
$$

The operators $E_{\lambda}(\varphi, \Delta) \in \mathscr{B}(\mathscr{H})$ are defined for $\Delta \in \mathscr{G}_{\lambda}(\varphi)$ in accordance with Lemma 5.1 by the sesquilinear forms

$$
\begin{aligned}
\left(E_{\lambda}(\varphi, \Delta) f, g\right)= & \lim _{\varepsilon \downarrow 0} \frac{1}{2 \pi i} \int\left(\left\{R\left(\varphi, \lambda+(\mu+i \varepsilon) e^{2 i \varphi}\right)\right.\right. \\
& \left.\left.-R\left(\varphi, \lambda+(\mu-i \varepsilon) e^{2 i \varphi}\right)\right\} f, g\right) d \mu \quad f, g \in \mathscr{H} .
\end{aligned}
$$

Lemma 5.3. For fixed $\varphi \neq 0$, the operators $E_{\lambda}(\varphi, \Delta)$ satisfy the following conditions :

$$
E_{\lambda_{i}}\left(\varphi, \Delta_{1}\right) E_{\lambda_{2}}\left(\varphi, \Delta_{2}\right)=\delta_{\lambda_{1}}^{\lambda_{2}} E_{\lambda_{1}}\left(\varphi, \Delta_{1} \cap \Delta_{2}\right) \quad \text { for } \quad \Delta_{i} \in \mathscr{G}_{\lambda_{i}}(\varphi), \quad i=1,2 .
$$

For any finite set $\left\{\Delta_{k}\right\}_{k=1}^{m}$ of pairwise disjoint sets in $\mathscr{G}_{\lambda}(\varphi)$,

$$
\begin{gathered}
E_{\lambda}\left(\varphi, \bigcup_{k=1}^{m} \Delta_{k}\right)=\sum_{k=1}^{m} E_{\lambda}\left(\varphi, \Delta_{k}\right), \\
E_{\lambda}(\varphi, \Delta) H \subseteq H E_{\lambda}(\varphi, \Delta) .
\end{gathered}
$$

Proof. This is similar to the proof of [8], Lemma 7.6, utilizing Lemmas 5.1, 2.14, 3.13 with simple modifications due to the presence of the term $\left\langle\phi_{D}^{i}(\bar{z})\right|$ in $Y_{D}^{i}(z)$.

Definition 5.4. For $\varphi \neq 0, \lambda=\lambda_{D}^{i} \in \Sigma_{2}, \Delta \in \mathscr{G}_{\lambda}(\varphi)$ we set

$$
\begin{aligned}
& \tilde{F}_{\lambda \pm}(\varphi, \Delta)=\chi_{\Delta}(\mu) T_{D}(\mu) Y_{\lambda \pm}\left(\varphi, \lambda+\mu e^{2 i \varphi}\right), \\
& F_{\lambda \pm}(\varphi, \Delta)=\tilde{F}_{\lambda \pm}(\varphi, \Delta) \mid E_{\lambda}(\varphi, \Delta) \mathscr{H} .
\end{aligned}
$$

For $\varphi=0$ and $\bar{\Delta}$ a compact subset of $\left(0, \lambda^{\prime}-\lambda\right) \backslash \tilde{\sigma}_{p}(H)$ we set

$$
\begin{aligned}
& \tilde{F}_{\lambda \pm}(\Delta)=\chi_{\Delta}(\mu) \sum_{D \in D_{E}} \sum_{\lambda_{D}^{i} \leqq \lambda} \oplus T_{D}^{i}(\lambda+\mu) Y_{D \pm}^{i}(\lambda+\mu), \\
& F_{\lambda \pm}(\Delta)=\tilde{F}_{\lambda \pm}(\Delta) \mid E(\lambda+\Delta) \mathscr{H} .
\end{aligned}
$$

We shall now establish the basic properties of the local inverse wave operators $F_{\lambda \pm}(\varphi, \Delta)$ associated with each channel for $\varphi \neq 0$ as given by (5.7), (5.8).

Lemma 5.5. The operators $\tilde{F}_{\lambda \pm}(\varphi, \Delta)$ are in $\mathscr{B}\left(\mathscr{H}, L^{2}\left(\Delta, h_{D}\right)\right)$, and

$$
\tilde{F}_{\lambda \pm}(\varphi, \Delta) E_{\lambda}(\varphi, \Delta)=\tilde{F}_{\lambda \pm}(\varphi, \Delta) .
$$

Proof. The first statement follows from Lemma 3.13 and [8], Lemma 1.3. Then it is proved as in [8], Lemma 7.10 that $E_{\lambda}(\varphi, \Delta)=0$ implies $\tilde{F}_{\lambda \pm}(\varphi, \Delta)=0$, using Lemmas 3.10, 5.1, and 5.3 and [8], Lemma 1.3, and 5.11 follows. 
Theorem 5.6. The operators $F_{\lambda_{ \pm}}(\varphi, \Delta)$ are $1-1$ and bicontinuous from $E_{\lambda}(\varphi, \Delta) \mathscr{H}$ onto $L^{2}\left(\Delta, h_{D}\right)$. Moreover, for $\tilde{\Delta} \cong \Delta$ and $\hat{u} \in L^{2}\left(\Delta, h_{D}\right)$

$$
F_{\lambda \pm}(\varphi, \Delta) E_{\lambda}(\varphi, \tilde{\Delta}) F_{\lambda \pm}^{-1}(\varphi, \Delta) u=\chi_{\hat{\Delta}} \hat{u} .
$$

Proof. Let $f \in E_{\lambda}(\varphi, \Delta) \mathscr{H}$. Then by Lemma 5.1 and (5.3)

$$
\begin{aligned}
\|f\| & =\sup _{\|g\|=1}\left|\left(F_{\lambda}(\varphi, \Delta) f, F_{\lambda}(-\varphi, \Delta) g\right)_{L^{2}\left(\Delta, h_{D}\right)}\right| \\
& \leqq\left\|F_{\lambda}(\varphi, \Delta) f\right\|_{L^{2}\left(\Delta, h_{D}\right)} \cdot\left\|F_{\lambda}(-\varphi, \Delta)\right\|_{\mathscr{B}\left(\mathscr{H}, L^{2}\left(\Delta, h_{D}\right)\right)} .
\end{aligned}
$$

This proves that $F_{\lambda}(\varphi, \Delta)$ is $1-1$ and $F_{\lambda}^{-1}$ is bounded. It is then proved as in [8], Lemma 7.12 that $\mathscr{R}\left(F_{\lambda \pm}(\varphi, \Delta)\right)$ is dense in $L^{2}\left(\Delta, h_{D}\right)$ and hence $F_{\lambda \pm}(\varphi, \Delta)$ is onto $L^{2}\left(\Delta, h_{D}\right)$.

Theorem 5.7. The operators $F_{\lambda \pm}(\Delta)$ defined by (5.9) and (5.10) are isometries from $E(\lambda+\Delta) \mathscr{H}$ onto $\sum_{D \in \mathscr{D}_{E}} \sum_{\lambda_{D}^{i} \leqq \lambda} \oplus L^{2}\left(\lambda-\lambda_{D}^{i}+\Delta, h_{D}\right)$.

There exist unique isometric operators $F_{ \pm}\left(\lambda, \lambda^{\prime}\right)$ from $E\left(\left(\lambda, \lambda^{\prime}\right) \backslash \sigma_{p}(H)\right) \mathscr{H}$ onto

$$
\left.\sum_{D \in \mathscr{D} E} \sum_{\lambda i_{D}^{i} \leqq \lambda} \oplus L^{2}\left(\lambda-\lambda_{D}^{i}, \lambda^{\prime}-\lambda_{D}^{i}\right), h_{D}\right)
$$

such that for $\bar{\Delta}$ a compact subset of $\left(0, \lambda^{\prime}-\lambda\right) \backslash \tilde{\sigma}_{p}(H)$,

$$
F_{ \pm}\left(\lambda, \lambda^{\prime}\right) \mid E(\lambda+\Delta) \mathscr{H}=F_{\lambda \pm}(\Delta) .
$$

For $\tilde{\Delta} \subseteq\left(0, \lambda^{\prime}-\lambda\right) \backslash \sigma_{p}(H)$ and

$$
\begin{gathered}
\hat{u}=\sum_{D \in \mathscr{D} E} \sum_{\lambda_{D}^{i} \leqq \lambda} \oplus u_{D}^{i}, \quad u_{D}^{i} \in L^{2}\left(\left(\lambda-\lambda_{D}^{i}, \lambda^{\prime}-\lambda_{D}^{i}\right), h_{D}\right), \\
F_{ \pm}\left(\lambda, \lambda^{\prime}\right) E(\lambda+\tilde{\Delta}) F_{ \pm}^{-1}\left(\lambda, \lambda^{\prime}\right) \hat{u}=\sum_{D \in \mathscr{D} E} \sum_{\lambda_{D}^{i} \leqq \lambda} \oplus \chi_{\lambda-\lambda_{D}^{i}+\tilde{\Delta}^{-} u_{D}^{i} .}
\end{gathered}
$$

Moreover,

$$
F_{ \pm}^{-1}\left(\lambda, \lambda^{\prime}\right) \hat{u}=\underset{t \rightarrow \pm \infty}{S-\lim _{t}} e^{i t H} J e^{-i t H_{1}} \hat{u}
$$

Proof. The first statement is proved, utilizing Lemma 4.4 and (5.2), in the same way as Theorem I of Kato [20], cf. [35], Theorem 1.6 and [8], Theorem 7.13. Since $\tilde{\sigma}_{p}(H) \backslash \sigma_{p}(H)$ consists of isolated points $\mu$ such that $E(\{\mu\})=0$, the operators $F_{ \pm}\left(\lambda, \lambda^{\prime}\right)$ defined on $\bigcup_{\Delta} E(\lambda+\Delta) \mathscr{H}$ by (5.14) immediately extend to isometries on $E\left(\left(\lambda, \lambda^{\prime}\right) \backslash \sigma_{p}(H)\right) \mathscr{H}$. The identity $(5.15)$ is proved for $\overline{\tilde{\Delta}} \cong\left(0, \lambda^{\prime}-\lambda\right) \backslash \tilde{\sigma}_{p}(H)$ as in [35], see also [8], and extended to $\tilde{\Delta} \cong\left(0, \lambda^{\prime}-\lambda\right) \backslash \sigma_{p}(H)$, using the isometric property of $F\left(\lambda, \lambda^{\prime}\right)$. The identification (5.16) of the operators $F^{-1}\left(\lambda, \lambda^{\prime}\right)$ with the wave operators defined in the time-dependent theory is proved in the same way as in [35], in fact the invariance principle holds.

Definition 5.8. For $\varphi \neq 0, \lambda=\lambda_{D}^{i} \in \Sigma_{2}, \Delta \in \mathscr{G}_{\lambda}(\varphi)$, the local wave operators

$$
W_{\lambda \pm}(\varphi, \Delta) \in \mathscr{B}\left(L^{2}\left(\Delta, h_{D}\right), E_{\lambda}(\varphi, \Delta) \mathscr{H}\right)
$$


are defined in accordance with Theorem 5.6 by

$$
W_{\lambda \pm}(\varphi, \Delta)=F_{\lambda \pm}^{-1}(\varphi, \Delta) .
$$

The local scattering operators $S_{\lambda}(\varphi, \Delta) \in \mathscr{B}\left(L^{2}\left(\Delta, h_{D}\right)\right)$ are defined by

$$
S_{\lambda}(\varphi, \Delta)=W_{\lambda+}^{-1}(\varphi, \Delta) W_{\lambda-}(\varphi, \Delta) .
$$

For $\varphi=0$ the wave operators

$$
W_{ \pm}\left(\lambda, \lambda^{\prime}\right) \in \mathscr{B}\left(\sum_{D \in \mathscr{D} E} \sum_{\lambda_{D}^{i} \leqq \lambda} \oplus L^{2}\left(\left(\lambda-\lambda_{D}^{i}, \lambda^{\prime}-\lambda_{D}^{i}\right), h_{D}\right), E\left(\left(\lambda, \lambda^{\prime}\right) \backslash \sigma_{p}(H)\right) \mathscr{H}\right)
$$

are defined in accordance with Theorem 5.7 by

$$
W_{ \pm}\left(\lambda, \lambda^{\prime}\right)=F_{ \pm}^{-1}\left(\lambda, \lambda^{\prime}\right)
$$

The scattering operators

$$
S\left(\lambda, \lambda^{\prime}\right) \in \mathscr{B}\left(\sum_{D \in \mathscr{D}} \sum_{\lambda_{D}^{i} \leqq \lambda} \oplus L^{2}\left(\left(\lambda-\lambda_{D}^{i}, \lambda^{\prime}-\lambda_{D}^{i}\right), h_{D}\right)\right)
$$

are defined by

$$
S\left(\lambda, \lambda^{\prime}\right)=W_{+}^{-1}\left(\lambda, \lambda^{\prime}\right) W_{-}\left(\lambda, \lambda^{\prime}\right) .
$$

It follows from Theorem 5.7 that $S\left(\lambda, \lambda^{\prime}\right)$ is a unitary operator on

$$
\sum_{D \in \mathscr{D}_{E}} \sum_{\lambda_{D}^{i} \leqq \lambda} \oplus L^{2}\left(\left(\lambda-\lambda_{D}^{i}, \lambda^{\prime}-\lambda_{D}^{i}\right), h_{D}\right),
$$

which commutes with $\sum_{D \in \mathscr{D}_{E}} \sum_{\lambda_{D}^{i} \leqq \lambda} \oplus \chi_{\lambda-\lambda_{D}^{i}+\Delta}(\mu)$ for $\Delta \subseteq\left(0, \lambda^{\prime}-\lambda\right)$.

It follows from Theorem 5.6 that $S_{\lambda}(\varphi, \Delta)$ is a bicontinuous isomorphism of $L^{2}\left(\Delta, h_{D}\right)$ which commutes with $\chi_{\Delta}(\mu)$ for $\tilde{\Delta} \subseteq \Delta$.

To obtain a representation of $S\left(\lambda, \lambda^{\prime}\right)$ and $S_{\lambda}(\varphi, \Delta)$ in terms of the scattering matrix, we need the following result.

Lemma 5.9. For $\varphi \neq 0, \lambda=\lambda_{D}^{i} \in \Sigma_{2}, \Delta \in G_{\lambda}(\varphi), u \in \mathscr{H}$ and $v \in H_{p_{D}}^{s}$,

$$
\begin{gathered}
\left(F_{\lambda \pm}(\varphi, \Delta) E_{\lambda}(\varphi, \Delta) u, \chi_{\Delta}(\mu) T_{D}(\mu) v\right)_{L^{2}\left(\Delta, h_{D}\right)}=e^{2 i \varphi}\left(E_{\lambda}(\varphi, \Delta) u,\left|\phi_{D}^{i}(-\varphi)\right\rangle v\right) \\
+\lim _{\varepsilon \downarrow 0} \frac{e^{2 i \varphi}}{2 \pi i}\left(\left\{R\left(\varphi, \lambda+(\mu+i \varepsilon) e^{2 i \varphi}\right)-R\left(\varphi, \lambda+(\mu-i \varepsilon) e^{2 i \varphi}\right)\right\} u,\right. \\
\left.W_{D}^{i}(-\varphi) r_{D}^{i}\left(-\varphi, \lambda+(\mu \pm i \varepsilon) e^{-2 i \varphi}\right) v\right)_{L^{2}(\Delta, \mathscr{H})} .
\end{gathered}
$$

For $\varphi=0, \lambda \in \Sigma_{2}, \lambda<E, \lambda+\Delta \leqq\left(\lambda, \lambda^{\prime}\right) \backslash \sigma_{p}(H), u \in \mathscr{H}$ and

$$
\begin{gathered}
v \in \sum_{D \in \mathscr{D}_{E}} \sum_{\lambda_{D}^{i} \leqq \lambda} \oplus H^{s}\left(R_{p_{D}}^{m}\right), \\
\left(F_{ \pm}\left(\lambda, \lambda^{\prime}\right) E(\Delta) u, \tilde{\chi}_{\Delta}(\mu) T(\lambda+\mu) v\right) \sum_{D \in \mathscr{D}_{E}} \sum_{\lambda_{D}^{i} \leqq \lambda} \oplus L^{2}\left(\Delta, h_{D}\right) \\
=\left(E(\Delta) u, \sum_{D \in \mathscr{D}_{E}} \sum_{\lambda_{D}^{i} \leqq \lambda}\left|\phi_{D}^{i}(-\varphi)\right\rangle v_{D}^{i}\right)+\lim _{\varepsilon \downarrow 0} \frac{1}{2 \pi i} \\
\cdot\left(\{R(\lambda+\mu+i \varepsilon)-R(\lambda+\mu-i \varepsilon)\} u, \sum_{D \in \mathscr{D}_{E}} \sum_{\lambda_{D}^{i} \leqq \lambda} W_{D}^{i}(-\varphi) r_{D}^{i}(-\varphi, \lambda+\mu \pm i \varepsilon) v_{D}^{i}\right),
\end{gathered}
$$


where $\tilde{\chi}_{\Delta}(\mu)=\sum_{D \in \mathscr{D}_{E}} \sum_{\lambda_{D}^{i} \leqq \lambda} \oplus \chi_{\Delta}(\mu)$ and $v=\sum_{D \in \mathscr{D}_{E}} \sum_{\lambda_{D} \leqq \lambda} \oplus v_{D}^{i}$.

Proof. This is proved in the same way as [8], Lemma 8.1 utilizing Lemmas 2.12-2.15, 4.2, and 5.5.

Theorem 5.10. For $\varphi \neq 0, \lambda=\lambda_{D}^{i} \in \Sigma_{2}, \Delta \in \mathscr{D}_{E}(\lambda)$, the local scattering operators $S_{\lambda}(\varphi, \Delta)$ and their inverses $S_{\lambda}^{-1}(\varphi, \Delta)$ have the following representations for $f \in L^{2}\left(\Delta, h_{D}\right)$ and a.e. $\mu \in \Delta$,

$$
\begin{gathered}
\left(S_{\lambda}(\varphi, \Delta) f\right)(\mu)=\chi_{\Delta}(\mu) \mathscr{S}_{\lambda}(\varphi, \mu) f(\mu), \\
\left(S_{\lambda}^{-1}(\varphi, \Delta) f\right)(\mu)=\chi_{\Delta}(\mu) \mathscr{S}_{\lambda}^{-1}(\varphi, \mu) f(\mu),
\end{gathered}
$$

where $\mathscr{S}_{\lambda}(\varphi, \mu)$ and its inverse $\mathscr{S}_{\lambda}^{-1}(\varphi, \mu)$ are defined by

$$
\begin{gathered}
\mathscr{S}_{\lambda}(\varphi, \mu)=1-2 \pi i e^{-2 i \varphi} T_{D}(\mu)\left[Y_{\lambda-}\left(-\varphi, \lambda+\mu e^{-2 i \varphi}\right) W_{D}^{i}(-\varphi)\right]^{*} T_{D}^{*}(\mu), \\
\mathscr{S}_{\lambda}^{-1}(\varphi, \mu)=1+2 \pi i e^{-2 i \varphi} T_{D}(\mu)\left[Y_{\lambda+}\left(-\varphi, \lambda+\mu e^{-2 i \varphi}\right) W_{D}^{i}(-\varphi)\right]^{*} T_{D}^{*}(\mu) .
\end{gathered}
$$

For fixed $\varphi_{(>)}^{<} 0$ the operators $\mathscr{S}_{\lambda}(\varphi, \mu)$ form a norm-continuous function of

$$
\mu \in \mathrm{R}^{+} \backslash\left\{\mu \mid \lambda+\mu e^{2 i \varphi} \in \tilde{\mathscr{R}}_{\lambda}\left(\overline{\mathscr{R}}_{\lambda}^{\prime}\right)\right\}
$$

with values in $\mathscr{B}\left(h_{D}\right)$, and $\mathscr{S}^{-1}(\varphi, \mu)$ a norm-continuous function of

$$
\mu \in \mathrm{R}^{+} \backslash\left\{\mu \mid \lambda+\mu e^{2 i \varphi} e \tilde{\mathscr{R}}_{\lambda}^{\prime}\left(\tilde{\mathscr{R}}_{\lambda}\right)\right\}
$$

with values in $\mathscr{B}\left(h_{D}\right)$. Moreover,

$$
1-\mathscr{S}_{\lambda}(\varphi, \mu) \in \mathscr{C}\left(h_{D}\right), \quad 1-\mathscr{S}_{\lambda}^{-1}(\varphi, \mu) \in \mathscr{C}\left(h_{D}\right) .
$$

For $\varphi=0$ the scattering operators $S\left(\lambda, \lambda^{\prime}\right)$ and their inverses $S^{-1}\left(\lambda, \lambda^{\prime}\right)$ have the following representations for

$$
f \in \sum_{D \in \mathscr{D}_{E}} \sum_{\lambda_{D}^{i} \leqq \lambda} \oplus L^{2}\left(\left(\lambda-\lambda_{D}^{i}, \lambda^{\prime}-\lambda_{D}^{i}\right), h_{D}\right)
$$

and a.e. $\mu \in\left(0, \lambda^{\prime}-\lambda\right)$,

$$
\begin{gathered}
\left(S\left(\lambda, \lambda^{\prime}\right) f\right)(\mu)=\tilde{\chi}_{\left(\lambda, \lambda^{\prime}\right)}(\mu) \mathscr{S}_{\lambda}(\mu) f(\mu), \\
\left(S^{-1}\left(\lambda, \lambda^{\prime}\right) f(\mu)=\tilde{\chi}_{\left(\lambda, \lambda^{\prime}\right)}(\mu) \mathscr{S}_{\lambda}^{-1}(\mu) f(\mu),\right.
\end{gathered}
$$

where the scattering matrix $\mathscr{S}_{\lambda}(\mu)$ and its inverse $\mathscr{S}_{\lambda}^{-1}(\mu)$ are defined by

$$
\begin{aligned}
\mathscr{S}_{\lambda}(\mu) & =1-2 \pi i T(\lambda+\mu) Y_{+}(\lambda+\mu) W T^{*}(\lambda+\mu), \\
\mathscr{S}_{\lambda}^{-1}(\mu) & =1+2 \pi i T(\lambda+\mu) Y_{-}(\lambda+\mu) W T^{*}(\lambda+\mu),
\end{aligned}
$$

where we have set $E=\lambda^{\prime}$ in (2.23).

The operator $\mathscr{S}_{\lambda}(\mu)$ is unitary on $\sum_{D, i} \oplus h_{D}$ and norm-continuous for $\mu \in\left(0, \lambda^{\prime}-\lambda\right) \backslash \tilde{\sigma}_{p}(H)$.

Moreover,

$$
1-\mathscr{S}_{\lambda}(\mu) \in \mathscr{C}\left(\sum_{D, i} \oplus h_{D}^{i}\right)
$$


and

$$
1-\mathscr{S}_{\lambda}^{-1}(\mu) \in \mathscr{C}\left(\sum_{D, i} \oplus h_{D}^{i}\right) .
$$

Proof. This is proved as [8], Lemma 8.4, utilizing Lemmas 4.2, 5.1, and 5.9.

We proceed to study the analyticity properties and limits for $\varphi \rightarrow 0$ of $\mathscr{S}_{\lambda}^{-1}\left(\varphi, \varrho^{2} / 2 m_{D}\right)$ and $\mathscr{S}_{\lambda}\left(\varphi, \varrho^{2} / 2 m\right)$, establishing the connection of these operators with the diagonal elements $\left[\mathscr{S}_{\lambda}\left(\varrho^{2} / 2 m_{D}\right)\right]_{\lambda, \lambda}$ and $\left[\mathscr{S}_{\lambda}^{-1}\left(\varrho^{2} / 2 m_{D}\right)\right]_{\lambda, \lambda}$ of the S-matrix
on the corresponding energy interval.

Theorem 5.11. For $\lambda=\lambda_{D}^{i} \in \Sigma_{2}$, the $\mathscr{B}\left(h_{D}\right)$-valued functions $\mathscr{S}_{\lambda}(z)=\mathscr{S}_{\lambda}\left(\varphi, \varrho^{2} / 2 m_{D}\right)$ and $\mathscr{S}_{\lambda}^{-1}(z)=\mathscr{S}_{\lambda}^{-1}\left(\varphi, \varrho^{2} / 2 m_{D}\right)$ are meromorphic for $z \in \mathcal{O} \backslash R^{+}$with poles at most at points of $\left(\tilde{\mathscr{R}}_{\lambda} \cup \overline{\mathscr{R}}_{\lambda}^{\prime}\right)^{\sim}$ and $\left(\overline{\mathscr{R}}_{\lambda}^{\prime} \cup \tilde{\mathscr{R}}_{\lambda}\right)^{\llcorner}$respectively.

Moreover,

$$
\begin{gathered}
\mathscr{S}_{\lambda}\left(\varrho e^{i \varphi}\right) \underset{\varphi \rightarrow 0_{-}}{\longrightarrow}\left(\left[\mathscr{S}^{-1}\left(\varrho^{2} / 2 m_{D}\right)\right]_{\lambda, \lambda}\right)^{*}, \\
\mathscr{S}_{\lambda}^{-1}\left(\varrho e^{i \varphi}\right) \underset{\varphi \rightarrow 0_{+}}{\longrightarrow}\left(\left[\mathscr{S}_{\lambda}\left(\varrho^{2} / 2 m_{D}\right)\right]_{\lambda, \lambda}\right)^{*}
\end{gathered}
$$

in the uniform operator topology of $\mathscr{B}\left(h_{D}\right)$, uniformly for $\varrho$ in any compact subset of $\left\{\left(\lambda, \lambda^{\prime}\right) \backslash \tilde{\sigma}_{p}(H)\right\}^{2}$.

Proof. By (4.1) and the identity

$$
\varrho^{m / 2} \gamma_{D}(\varrho)=\gamma_{D}(1) U_{D}(\varrho)
$$

we have, setting $\mu=\varrho^{2} / 2 m_{D}$

$$
T_{D}(\mu)=m_{D}^{1 / 2} \varrho^{-1} \gamma_{D}(1) U_{D}(\varrho) .
$$

Introducing (5.37) in (5.25) and (5.26), we obtain for $\varphi \neq 0$, taking adjoints and replacing $\varphi$ by $-\varphi$,

$$
\begin{aligned}
\mathscr{S}_{\lambda}^{*}(\bar{z}) & =1+2 \pi i z^{-2} m_{D} \gamma_{D}(1) U_{D}(\varrho) Y_{D-}^{i}\left(\varphi, \lambda+\frac{z^{2}}{2 m_{D}}\right) W_{D}^{i}(\varphi) U_{D}^{*}(\varrho) \gamma_{D}^{*}(1) \\
& =1+2 \pi i z^{-2} m_{D} \gamma_{D}(1) Y_{D-}^{i}\left(z, \lambda+\frac{z^{2}}{2 m_{D}}\right) W_{D}^{i}(z) \gamma_{D}^{*}(1) .
\end{aligned}
$$

and similarly

$$
\mathscr{S}_{\lambda}^{-1 *(\bar{z})}=1-2 \pi i z^{-2} m_{D} \gamma_{D}(1) Y_{D+}^{i}\left(z, \lambda+\frac{z^{2}}{2 m_{D}}\right) W_{D}^{i}(z) \gamma_{D}^{*}(1) .
$$

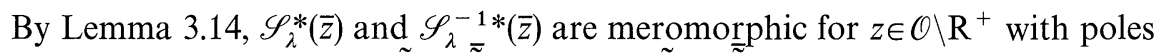
at most at points of $\left(\tilde{\mathscr{R}}_{\lambda}^{\prime} \cup \tilde{\mathscr{R}}_{\lambda}\right)^{\llcorner}$and $\left(\tilde{\mathscr{R}}_{\lambda} \cup \tilde{\tilde{R}}_{\lambda}^{\prime}\right)^{\llcorner}$respectively, and for $\lambda+\frac{\varrho^{2}}{2 m_{D}} \in\left(\lambda, \lambda^{\prime}\right) \backslash \tilde{\sigma}_{p}$

$$
\begin{gathered}
\lim _{\varphi \rightarrow 0_{+}} \mathscr{S}_{\lambda}^{*}(\bar{z})=1+2 \pi i \varrho^{-2} m_{D} \gamma_{D}(1) Y_{D-}^{i}\left(\varrho, \lambda+\frac{\varrho^{2}}{2 m_{D}}\right) W_{D}^{i}(\varrho) \gamma_{D}^{*}(1), \\
\lim _{\varphi \rightarrow 0_{-}} \mathscr{S}_{\lambda}^{-1 *}(\bar{z})=1-2 \pi i \varrho^{-2} m_{D} \gamma_{D}(1) Y_{D+}^{i}\left(\varrho, \lambda+\frac{\varrho^{2}}{2 m_{D}}\right) W_{D}^{i}(\varrho) \gamma_{D}^{*}(1) .
\end{gathered}
$$


By (5.30), (5.31), and (5.37), the right hand sides of (5.40) and (5.41) coincide with $\left[\mathscr{S}_{\lambda}^{-1}\left(\varrho^{2} / 2 m_{D}\right)\right]_{\lambda, \lambda}$ and $\left[\mathscr{S}_{\lambda}\left(\varrho^{2} / 2 m_{D}\right)\right]_{\lambda, \lambda}$ respectively, and the lemma is proved.

Corollary 5.12. There exist closed null sets $N_{\lambda \pm} C\left(\lambda, \lambda^{\prime}\right)$, such that $\left[\mathscr{S}_{\lambda}^{(-1)}\left(\varrho^{2} / 2 m_{D}\right)\right]_{\lambda, \lambda}$ is invertible for $\varrho \in\left\{\left(\lambda, \lambda^{\prime}\right) \backslash N_{\lambda_{( \pm} \pm}\right\}^{\nu}$, and

$$
\begin{gathered}
\lim _{\varphi \rightarrow 0_{-}} \mathscr{S}_{\lambda}^{*}(\bar{z})=\left(\left[\mathscr{S}_{\lambda}\left(\varrho^{2} / 2 m_{D}\right)\right]_{\lambda, \lambda}\right)^{-1}, \\
\lim _{\varphi \rightarrow 0_{+}} \mathscr{S}_{\lambda}^{-1} *(\bar{z})=\left(\left[\mathscr{S}_{\lambda}^{-1}\left(\varrho^{2} / 2 m_{D}\right)\right]_{\lambda, \lambda}\right)^{-1}
\end{gathered}
$$

in the uniform operator topology of $\mathscr{B}\left(h_{D}\right)$, uniformly for $\varrho$ in any compact subset of $\left\{\left(\lambda, \lambda^{\prime}\right) \backslash N_{\lambda_{1}+ \pm}\right\}^{2}$.

Proof. This follows from Theorem 5.11, (5.27) and a result of Kuroda [21].

We finally turn to the question of the connection between resolvent resonances and poles of the S-matrix.

The treatment of this problem is more complicated than in the two-body case (see [7]), partly because of the inherent difficulty due to the possibility of embedded eigenvalues and partly because of the possibility of spurious poles of $\left(1-I_{r \pm}\left(z, \lambda+\frac{z^{2}}{2 m_{D}}\right)\right)^{-1}$ due to the method, using the Weinberg equation.

We shall not give a complete answer here, but prove the following results which seem to cover most cases.

Theorem 5.13. Let $\lambda=\lambda_{D}^{i} \in \Sigma_{2}$, and $\kappa=\lambda+\frac{z^{2}}{2 m_{D}} \in \mathscr{R}_{\lambda} \mid \tilde{\mathscr{R}}_{\lambda}^{\prime}\left(\mathscr{R}_{\lambda}^{\prime} \backslash \tilde{\mathscr{R}}_{\lambda}\right)$ for $\varphi<0$ and $\lambda \in \overline{\mathscr{R}}_{\lambda}^{\prime} \backslash \overline{\mathscr{R}}_{\lambda}\left(\overline{\mathscr{R}}_{\lambda} \backslash \overline{\tilde{\mathscr{R}}}_{\lambda}^{\prime}\right)$ for $\varphi>0$, and define the operators $T_{\left.\kappa_{(} \pm\right)}$and $Z_{\Lambda_{(} \pm}$, by

$$
\begin{aligned}
& \sigma=T_{\left.h^{+}{ }_{-}\right)} \Omega=2 \pi i z^{-2} \gamma_{D}(1) E_{\lambda} \Omega, \quad \Omega=(u, \tau) \in \mathscr{N}\left(G_{\left.\lambda_{( \pm}{ }^{ \pm}\right)}(z, \kappa),\right. \\
& \Omega=Z_{\kappa_{(-)} \sigma} \sigma=-m_{D} Y_{\lambda_{(\mp)}}(z, \kappa) W_{D}^{i}(z) \gamma_{D}^{*}(1) \sigma,
\end{aligned}
$$

where $E_{\lambda} \Omega=\tau$.

The operator $T_{\left.\kappa_{(}{ }^{+}\right)}$is an isomorphism from $\mathscr{N}\left(G_{\left({ }^{ \pm}\right)}(z, \kappa)\right)$ onto $\mathscr{N}\left(\mathscr{S}_{\lambda}^{*(-1)}(\bar{z})\right)$ with the inverse $Z_{\kappa_{( \pm)}}$.

Proof. We consider $\left(T_{\kappa+}, Z_{\kappa+}\right)$ for $\varphi<0$, the proof in the other cases is similar. For brevity we set $T_{\kappa+}=T_{\kappa}, Z_{\kappa+}=Z_{\kappa}$ and $G_{ \pm}=G_{\lambda \pm}, Y_{ \pm}=Y_{\lambda \pm}, R_{1 \lambda \pm}=R_{1 \pm}, W_{\lambda}=W$.

1) $W_{D}^{i}(z) 2 \pi i m_{D} z^{-2} \gamma_{D}^{*}(1) \gamma_{D}(1) E_{\lambda}=G_{+}(z, \kappa)-G_{-}(z, \kappa)$.

By Lemma 4.2

$$
\begin{aligned}
W_{D}^{i}(z) 2 \pi i m_{D} z^{-2} \gamma_{D}^{*}(1) \gamma_{D}(1) E_{\lambda} & =W_{D}^{i}(z)\left\{r_{D+}^{i}(z, \kappa)-r_{D-}^{i}(z, \kappa)\right\} E_{\lambda} \\
& =W(z)\left\{R_{1+}(z, \kappa)-R_{1-}(z, \kappa)\right\} \\
& =G_{+}(z, \kappa)-G_{-}(z, \kappa),
\end{aligned}
$$

since $R_{0}(z, \xi)$ is regular at $\zeta=\kappa$.

2) $Z_{\kappa}$ maps $\mathscr{N}\left(\mathscr{S}_{\lambda}^{*}(\bar{z})\right.$ one-to-one into $\tilde{\mathcal{N}}\left(G_{+}(z, \kappa)\right)$, and $T_{\kappa} Z_{\kappa}=1$.

Let $\sigma \in \mathscr{N}\left(\mathscr{S}_{\lambda}^{*}(\bar{z})\right)$ and $\Omega=Z_{\kappa} \sigma$, i.e. by $(5.38)$

$$
\sigma+2 \pi i m_{D} z^{-2} \gamma_{D}(1) E_{\lambda} Y_{-}(z, \kappa) W_{D}^{i}(z) \gamma_{D}^{*}(1) \sigma=0
$$


Applying $Z_{\lambda}$ to (5.46), we get by 1 )

$$
\begin{gathered}
\Omega-m_{D} Y_{-}(z, \kappa) W_{D}^{i}(z) 2 \pi i m_{D} z^{-2} \gamma_{D}^{*}(1) \gamma_{D}(1) E_{\lambda} Y_{-}(z, \kappa) W_{D}^{i}(z) \gamma_{D}^{*}(1) \sigma \\
=\Omega+Y_{-}(z, \kappa)\left(G_{+}(z, \kappa)-G_{-}(z, \kappa)\right) \Omega=0 .
\end{gathered}
$$

Applying $G_{-}(z, \kappa)$ to (5.47), we get by (3.9)

$$
G_{+}(z, \kappa) \Omega=0 .
$$

From (5.44)-(5.46) follows

$$
T_{\kappa} Z_{\kappa} \sigma=\sigma .
$$

Also, if $Z_{\kappa} \sigma \in \mathscr{N}\left(J_{\lambda}(z) R_{1+}(z, \kappa)\right)$, then by Lemma $4.3 T_{\kappa} Z_{\kappa} \sigma=0$, so by (5.49) $\sigma=0$, and 2) is proved.

3) $T_{\kappa}$ maps $\tilde{N}\left(G_{+}(z, \kappa)\right)$ one-to-one into $\mathscr{N}\left(\mathscr{S}_{\lambda}^{*}(\bar{z})\right)$, and $Z_{\kappa} T_{\kappa}=1$.

Let $\Omega \in \mathscr{N}\left(G_{+}(z, \kappa)\right)$, and let $\sigma=T_{\kappa} \Omega$. Then by 1$)$ and (4.11) of Lemma 4.4

$$
\begin{aligned}
S_{\lambda}^{*}(\bar{z}) \sigma & =\sigma+2 \pi i m_{D} z^{-2} \gamma_{D}(1) E_{\lambda} Y_{-}(z, \kappa) W_{D}^{i}(z) 2 \pi i z^{-2} \gamma_{D}^{*}(1) \gamma_{D}(1) E_{\lambda} \Omega \\
& =\sigma+2 \pi i z^{-2} \gamma_{D}(1) E_{\lambda} Y_{-}(z, \kappa)\left\{G_{+}(z, \kappa)-G_{-}(z, \kappa)\right\} \Omega \\
& =\sigma-2 \pi i z^{-2} \gamma_{D}(1) E_{\lambda} Y_{-}(z, \kappa) G_{-}(z, \kappa) \Omega \\
& =\sigma-2 \pi i z^{-2} \gamma_{D}(1) \Omega=0 .
\end{aligned}
$$

By (4.9) of Lemma 4.3, $T_{\kappa} \Omega=0$ for $\Omega \in \mathscr{N}\left(J(z) R_{1 \lambda+}(z, \kappa)\right)$, so $T_{\kappa}$ maps $\tilde{\mathscr{N}}\left(G_{+}(z, \kappa)\right)$ into $\mathscr{N}\left(\mathscr{S}_{\lambda}^{*}(\bar{z})\right)$.

By Lemma 4.6 , if $\Omega \in \tilde{\mathscr{N}}\left(G_{+}(z, \kappa)\right)$ and $T_{\kappa} \Omega=0$, then $\Omega \in \tilde{\mathscr{N}}\left(G_{-}(z, \kappa)\right)$. This by Lemmas 4.6 and 4.7 and the assumption that $\kappa \notin \mathscr{R}_{\lambda}^{\prime}$ implies that $\Omega=0$, so $T_{\kappa}$ is oneto-one.

By 2), $T_{\kappa}\left(Z_{\kappa} T_{\kappa} \Omega\right)=T_{\kappa} \Omega$ and hence $Z_{\kappa} T_{\kappa} \Omega=\Omega$, and 3) and thereby the theorem is proved.

We now consider the case, where $\kappa \in \mathscr{R}_{\lambda} \cap \tilde{\mathscr{R}}_{\lambda}^{\prime}$ for $\varphi<0\left(\lambda \in \overline{\mathscr{R}}_{\lambda}^{\prime} \cap \overline{\tilde{\mathscr{R}}}_{\lambda}\right.$ for $\left.\varphi>0\right)$, but $Y_{( \pm)}\left(\zeta, \lambda+\frac{\varrho^{2}}{2 m_{D}}\right)$ has a simple pole at $\zeta=\left(2 m_{D}(\kappa-\lambda)\right)^{1 / 2}$ and $\mathcal{N}\left(G_{(\overline{+})}(z, \kappa)\right)$ corresponds to an eigenspace of $\mathscr{N}(H(z)-\kappa)$. We first make the following observation.

Lemma 5.14. Assume that for $\varphi_{(\lessgtr) 0}$

$$
\gamma_{D}(1) E_{\lambda} \Omega=0 \text { for all } \Omega \in \mathscr{N}\left(G_{(\overline{+})}(z, \kappa)\right)
$$

and that $Y_{(\overline{+})}\left(\zeta, \lambda+\frac{\zeta^{2}}{2 m_{D}}\right)$ has a pole of order 1 at $\zeta=z$, i.e. for $\zeta$ near $z$

$$
Y_{(\overline{+})}\left(\zeta, \lambda+\frac{\zeta^{2}}{2 m_{D}}\right)=\frac{A_{(\overline{+})}}{\zeta-z}+\tilde{Y}_{(\bar{\mp})}\left(\zeta, \lambda+\frac{\zeta^{2}}{2 m_{D}}\right) \text {, }
$$

where $A_{\left.\overline{+}_{+}\right)} \in \mathscr{B}\left(H^{s}, \tilde{H}_{\lambda}^{s}\right)$ and $\tilde{Y}_{\left.\overline{(}_{+}\right)}\left(\zeta, \lambda+\frac{\zeta^{2}}{2 m_{D}}\right)$ is regular at $\zeta=z$.

Then $\mathscr{S}_{\lambda}^{*(-1)}(\bar{\zeta})$ is regular at $\zeta=z$, and

$$
\mathscr{S}_{\lambda}^{*(-1)}(\bar{z})=1_{( \pm)} 2 \pi i m_{D} z^{-2} \gamma_{D}(1) E_{\lambda} \tilde{Y}_{(\overline{(})}(z, \kappa) W_{D}^{i}(z) \gamma_{D}^{*}(1) \text {. }
$$




\section{Moreover}

$$
G_{(\mp)}(z, \kappa) \tilde{Y}_{(\mp)}(z, \kappa)=I .
$$

Proof. We consider $Y_{-}$for $\varphi<0$. By Lemma 3.11 and (5.52), for $\zeta$ near $z$

$$
\frac{G_{-}\left(\zeta, \lambda+\frac{\zeta^{2}}{2 m_{D}}\right) A_{-}}{\zeta-z}+G_{-}\left(\zeta, \lambda+\frac{\zeta^{2}}{2 m_{D}}\right) \tilde{Y}_{-}\left(\zeta, \lambda+\frac{\zeta^{2}}{2 m_{D}}\right)=I .
$$

This implies (5.54) and $G_{-}(z, \kappa) A_{-}=0$, hence by (5.51)

$$
\gamma_{D}(1) E_{\lambda} A_{-}=0 \text {. }
$$

For $\zeta$ near $z$

$$
\mathscr{S}_{\lambda}^{*}(\bar{\zeta})=1+2 \pi i m_{D} \zeta^{-2} \gamma_{D}(1) E_{\lambda} Y_{-}\left(\zeta, \lambda+\frac{\zeta^{2}}{2 m_{D}}\right) W(\zeta) \gamma_{D}^{*}(1) .
$$

By (5.52) and (5.56) $\mathscr{S}_{\lambda}^{*}(\bar{\zeta})$ is regular at $\zeta=z$, and $\mathscr{S}_{\lambda}^{*}(\bar{z})$ is given by (5.53).

Theorem 5.15. Assume that (5.51) and (5.52) hold. Let $T_{\left.\kappa_{(} \pm\right)}$and $Z_{\kappa_{(-)}}$be defined by

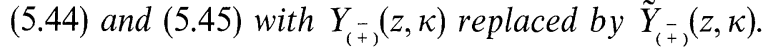

The operator $T_{\left.\kappa_{(}{ }^{ \pm}\right)}$induces an isomorphism from

$$
\tilde{\mathscr{N}}\left(G_{( \pm,}(z, \kappa)\right) / \tilde{\mathscr{N}}_{0}\left(G_{( \pm,}(z, \kappa)\right)
$$

onto $\mathscr{N}\left(\mathscr{S}_{\lambda}^{*(-1)}(\bar{z})\right)$ with the inverse $\tilde{Z}_{\left.\kappa_{(}{ }^{ \pm}\right)}$induced by $Z_{\left.\kappa_{(}{ }^{ \pm}\right)}$

Proof. This is proved as Theorem 5.13, replacing $Y_{\left(_{+}\right)}(z, \kappa)$ by $\tilde{Y}_{\left(_{+}\right)}(z, \kappa)$ and utilizing Lemma 5.14.

Remark 5.16. Under the assumption of Theorem 5.15 we obtain from Lemma 4.6 and Theorem 5.15 a decomposition

$$
\tilde{\mathcal{N}}\left(G_{+}(z, \kappa)\right)=\tilde{\mathscr{N}}_{0}\left(G_{+}(z, \kappa)\right) \dot{+} \tilde{\mathcal{N}}\left(G_{+}(z, \kappa)\right) / \tilde{\mathscr{N}}_{0}\left(G_{+}(z, \kappa)\right)
$$

such that

$$
\tilde{\mathscr{N}}_{0}\left(G_{+}(z, \kappa)\right)=\tilde{\mathscr{N}}_{0}\left(G_{-}(z, \kappa)\right) \text { is isomorphic to } \mathscr{N}(H(z)-\kappa)
$$

and

$$
\tilde{\mathcal{N}}\left(G_{+}(z, \kappa)\right) / \tilde{\mathscr{N}}_{0}\left(G_{+}(z, \kappa)\right) \text { is isomorphic to } \mathscr{N}\left(\mathscr{S}_{\lambda}^{*}(\bar{z})\right)
$$

and similarly for $\overline{\mathcal{N}}\left(G_{-}(z, \kappa)\right)$.

Theorem 5.17. 1) Let $\kappa=\lambda+\frac{z^{2}}{2 m_{D}}$, and suppose that

$$
\kappa \notin \tilde{\mathscr{R}}_{\lambda}^{\prime} \cup \overline{\tilde{\mathscr{R}}}_{\lambda}\left(\tilde{\mathscr{R}}_{\lambda} \cup \overline{\tilde{\mathscr{R}}}_{\lambda}^{\prime}\right) .
$$

Then $\mathscr{S}_{\lambda}^{(-1)}(\zeta)$ has a pole at $\zeta=z$ if and only if $\kappa \in \mathscr{R}_{\lambda} \cup \overline{\mathscr{R}}_{\lambda}^{\prime}\left(\mathscr{R}_{\lambda}^{\prime} \cup \overline{\mathscr{R}}_{\lambda}\right)$.

2) Suppose that (5.51) and (5.52) hold. 
Then $\mathscr{S}_{\lambda}^{(-1)}(\zeta)$ has a pole at $\zeta=z$ if and only if

$$
\kappa \in \mathscr{R}_{\lambda} \cup \overline{\mathscr{R}}_{\lambda}^{\prime}\left(\mathscr{R}_{\lambda}^{\prime} \cup \overline{\mathscr{R}}_{\lambda}\right) \quad \text { and } \quad \tilde{\mathscr{N}}\left(G_{( \pm)}(z, \kappa)\right) / \tilde{\mathscr{N}}_{0}\left(G_{\left({ }^{ \pm}\right.},(z, \kappa)\right) \neq\{0\} \text {. }
$$

Proof. We consider the case of $\mathscr{S}_{\lambda}(\zeta)$ for $\varphi<0$, the other cases are similar.

1) By Lemmas 4.6 and 4.7 and Theorems 5.11 and 5.13, the following statements are equivalent.

a) $\kappa \in \mathscr{R}_{\lambda}$;

b) $\bar{\kappa} \in \overline{\mathscr{R}}_{\lambda}$;

c) $\tilde{\mathscr{N}}\left(G_{-}(\bar{z}, \bar{\kappa})\right) \neq\{0\}$;

d) $\mathscr{N}\left(\mathscr{S}^{*-1}(z)\right) \neq\{0\}$;

e) $\bar{z}$ is a pole of $\mathscr{S}_{\lambda}^{*}(\bar{\zeta})$;

f) $z$ is a pole of $\mathscr{S}_{\lambda}(\zeta)$.

2) By Lemmas 4.6, 4.7, and 5.14 and Theorems 5.11 and 5.15, the following statements are equivalent.
a) $\kappa \in \mathscr{R}_{\lambda}$ and $\tilde{\mathcal{N}}\left(G_{+}(z, \kappa)\right) / \tilde{\mathscr{N}}_{0}\left(G_{+}(z, \kappa)\right) \neq\{0\}$;
b) $\bar{\kappa} \in \overline{\mathscr{R}}_{\lambda}$ and $\tilde{\mathscr{N}}\left(G_{-}(\bar{z}, \bar{\kappa})\right) / \tilde{\mathscr{N}}_{0}\left(G_{-}(\bar{z}, \bar{\kappa})\right) \neq\{0\}$;
c) $\mathscr{N}\left(\mathscr{S}_{\lambda}^{*-1}(z)\right) \neq\{0\}$;
d) $\bar{z}$ is a pole of $\mathscr{S}_{\lambda}^{*}(\bar{\zeta})$;
e) $z$ is a pole of $\mathscr{S}_{\lambda}(\zeta)$.

\section{Degenerate Thresholds}

In this section we briefly indicate the extension of the above results to the general case, where A3 is not satisfied, as it happens when there are symmetries in the system. We now assume that A1, A2 hold and moreover that $m_{D_{1}}=m_{D_{2}}$ if $\lambda_{D_{1}}^{i}=\lambda_{D_{2}}^{j}$ as it is the case if this coincidense is due to permutation symmetry. In Sect. 1, for $D=\left\{C_{1}, C_{2}\right\}, i=1 \ldots n(D)$, let

$$
d_{D}^{i}=\operatorname{dim} \mathscr{N}\left(H^{C_{1}}+H^{C_{2}}-\lambda_{D}^{i}\right),
$$

and choose in accordance with Lemma $2.10(2) \phi_{D}^{i j}(z), j=1 \ldots d_{D}^{i}$, such that $\left\{\phi_{D}^{i j}(z)\right\}$ is a basis of $\mathscr{N}\left(H^{C_{1}}+H^{C_{2}}-\lambda_{D}^{i}\right)$ and $\phi_{D}^{i j}(z)$ is analytic for $z \in \mathcal{O}$ for each $j$, and such that $\left(\phi_{D}^{i j}(z), \phi_{D}^{i k}(\bar{z})\right)=\delta_{j}^{k}$ for $z \in \mathcal{O}$. Set

$$
P_{D}^{i}(z)=\sum_{j=1}^{d_{D}^{i}}\left|\phi_{D}^{i j}(z)\right\rangle\left\langle\phi_{D}^{i j}(\bar{z})\right| .
$$

Then $P_{D}^{E}(z)$ and $R_{D}^{E}(z, \zeta)$ are defined as before, and the definitions of the various spaces and operators are modified in an obvious way, replacing $i$ by $i j$ and $\sum_{i=1}^{n(D)}$ by $\sum_{i=1}^{n(D)} \sum_{j=1}^{d_{D}^{i}}$ with $r_{D}^{i j}=r_{D}^{i}$ for $j=1 \ldots n_{D}^{i}$. Thus, $X$ now has the components $\left(X_{E},\left\{X_{D}^{i j}\right\}\right)$, $D \in \mathscr{D}_{E}, i=1 \ldots n(D), j=1 \ldots d_{D}^{i}$, for $\lambda=\lambda_{D}^{i}, X_{\lambda}$ has the components $\left(X_{E},\left\{X_{D}^{i j}\right\}\right)$, $j=1 \ldots d_{D}^{i}$.

The results of Sects. 2-4 are now extended accordingly in a rather obvious way. The proof of Lemma 4.3 in the general case requires an additional argument, for which we refer to [8]. 
The local inverse wave operators of Sect. 5 are constructed as before, but now the operators $F_{\lambda \pm}(\varphi, \Delta)$ for $\varphi \neq 0$ map onto

$$
\sum_{\lambda i_{D}^{i}=\lambda} \sum_{k=1}^{d_{D}^{i}} \oplus L^{2}\left(\Delta, h_{D}\right) .
$$

The basic properties of the inverse wave operators are derived in the same way, utilizing the generalized Lemma 4.3.

The scattering matrices $\mathscr{S}_{\lambda}(\varphi, \mu)$ and their inverses $\mathscr{S}_{\lambda}^{-1}(\varphi, \mu)$ in $\mathscr{B}\left(h_{D}\right)$ introduced in Theorem 5.10 are replaced by operators in

$$
\mathscr{B}\left(\sum_{\lambda_{D}=\lambda} \sum_{k=1}^{d_{D}^{i}} \oplus h_{D}^{(k)}\right),
$$

whose matrix elements can be written in the following form, utilizing (4.4) and the assumption $m_{D_{1}}=m_{D_{2}}$, for $\lambda_{D_{1}}^{i}=\lambda_{D_{2}}^{j}=\lambda, k=1 \ldots d_{D_{1}}^{i}, l=1 \ldots d_{D_{2}}^{j}$, setting $\mu=\varrho^{2} / 2 m_{D}$,

$$
\begin{array}{r}
\mathscr{S}_{\lambda_{\bar{D}}^{i}, \lambda_{D}^{J}}^{k, l}(\bar{z})=1+2 \pi i z^{-2} m_{D_{2}} \gamma_{D_{2}}(1) Y_{D_{2}}^{j 1}-\left(z, \lambda+\frac{z^{2}}{2 m_{D_{2}}}\right) W_{D_{1}}^{i k}(z) \gamma_{D_{1}}^{*}(1), \\
\left(\mathscr{S}^{-1}\right)_{\lambda_{D}^{k}, \lambda_{D}^{\prime}, \lambda_{D}^{j}}^{k, l}(\bar{z})=1-2 \pi i z^{-2} m_{D_{2} \gamma_{D_{2}}}(1) Y_{D_{2}+}^{j 1}\left(z, \lambda+\frac{z^{2}}{2 m_{D_{2}}}\right) W_{D_{1}}^{i k}(z) \gamma_{D_{1}}^{*}(1) .
\end{array}
$$

It then follows from Lemma 3.15, that each element of the matrices $\mathscr{S}_{\lambda}^{*}(\bar{z})$ and

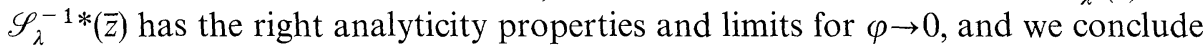
that Theorem 5.11 and Corollary 5.12 hold with $\mathscr{S}_{\lambda}(z)$ and $\mathscr{S}_{\lambda}^{-1}(z)$ replaced by the above defined matrices of operators and $\left[\mathscr{S}_{\lambda}\left(\varrho^{2} / 2 m_{D}\right)\right]_{\lambda, \lambda}$ and $\left[\mathscr{S}_{\lambda}^{-1}\left(\varrho^{2} / 2 \mathrm{~m}_{D}\right)\right]_{\lambda, \lambda}$ replaced by the partial scattering matrices related to the threshold $\lambda$, with the elements

$$
\begin{aligned}
& \mathscr{S}_{\lambda \lambda_{D}^{\prime}, \lambda D_{2}}^{k, l}\left(\varrho^{2} / 2 m_{D_{2}}\right)=1-2 \pi i m_{D_{2}} \gamma_{D_{2}}(1) Y_{D_{2}}^{j l}\left(\varrho, \lambda+\varrho^{2} / 2 m_{D_{2}}\right) W_{D_{1}}^{i k}(\varrho) \gamma_{D_{1}}^{*}(1), \\
& \left(\mathscr{S}^{-1}\right)_{\lambda_{D}^{k}, \lambda D_{2}^{\prime}}^{k, l}\left(\varrho^{2} / 2 m_{D_{2}}\right)=1+2 \pi i m_{D_{2}} \gamma_{D_{2}}(1) Y_{D}^{j l}\left(\varrho, \lambda+\varrho^{2} / 2 m_{D_{2}}\right) W_{D_{1}}^{i k}(\varrho) \gamma_{D_{1}}^{*}(1) \text {. }
\end{aligned}
$$

Theorem 5.13 holds with $T_{\kappa \pm}$ and $Z_{\kappa \pm}$ defined by

$$
\begin{aligned}
& T_{\left.\kappa_{(}+{ }_{-}\right)} \Omega=2 \pi i z^{-2} \sum_{\lambda_{D}^{i}=\lambda} \sum_{k=1}^{d_{D}^{i}} \oplus \gamma_{D}(1) E_{D}^{i k} \Omega, \quad \Omega \in \mathscr{N}\left(G_{\left( \pm^{ \pm}\right)}(z, \kappa)\right) \\
& Z_{\kappa_{(} \pm}\left(\sum_{\lambda_{D}^{i}=\lambda} \sum_{k=1}^{d_{D}^{i}} \oplus \sigma_{D}^{i k}\right)=-Y_{(\mp)}(z, \kappa) \sum_{\lambda_{D}^{i}=\lambda} \sum_{k=1}^{d_{D}^{i}} m_{D} W_{D}^{i k}(z) \gamma_{D}^{*}(1) \sigma_{D}^{i k} \\
& \sum_{\lambda=\lambda} \sum_{k=1}^{d_{D}^{i}} \oplus \sigma_{D}^{i k} \in \mathscr{N}\left(\mathscr{S}_{\lambda}^{*(-1)}(\bar{\kappa})\right)
\end{aligned}
$$

The proof is similar, utilizing the previous modifications. In Lemma 5.14, (5.51) is replaced by the condition

$$
T_{\left.\kappa_{(+)}\right)} \Omega=0 \text { for all } \Omega \in \mathscr{N}\left(G_{\kappa_{(+)}}(z, \kappa)\right.
$$

and (5.52) is assumed. Then (5.53) holds in the form of (6.2) and (6.3) with $Y$ replaced by $\tilde{Y}$, and (5.54) holds in the same form.

Theorem 5.15 is then generalized in the same way as Theorem 5.13, Remark 5.16 follows, and Theorem 5.17 is proved as before. 
In the case of permutation symmetry the problem of coincidence of thresholds could also be treated by reduction of $H(z)$ and $H_{0}(z)$ on subspaces of functions of a given symmetry (cf. [5]).

\section{References}

1. Agmon, S.: Ann. Scuola Norm. Super. Pisa, Cl. Scienze Ser. IV, Vol. II, 151-218 (1975)

2. Aguilar, F., Combes, J.M.: Commun. Math. Phys. 22, 269-279 (1971)

3. Babbitt, D., Balslev, E.: J. Funct. Anal. 18, 1-14 (1975)

4. Balslev, E., Combes, J.M.: Commun. Math. Phys. 22, 280-299 (1971)

5. Balslev, E.: Ann. Phys. 73, 49-107 (1972); Rep. Math. Phys. 5, 219-280; 393-413 (1974)

6. Balslev, E. : Commun. Math. Phys. 52, 127-146 (1977)

7. Balslev, E.: J. Funct. Anal. 29, 375-396 (1978)

8. Balslev, E.: Aarhus University, preprint no. $26(1978 / 79)$

9. Combes, J.M. : Nuovo Cimento 64A, 111-144 (1969)

10. Combes, J.M. : Commun. Math. Phys. 12, 283-295 (1969)

11. Combes, J.M., Thomas, L.E.: Commun. Math. Phys. 34, 251-270 (1973)

12. Enss, V.: Commun. Math. Phys. 65, 151-165 (1979)

13. Hagedorn, G.A.: Commun. Math. Phys. 65, 181-188 (1979)

14. Hepp, K.: Helv. Phys. Acta 42, 425-458 (1969)

15. Howland, J.S.: J. Funct. Anal. 22, 250-282 (1976)

16. Hunziker, M.: Helv. Phys. Acta 39, 451-462 (1966)

17. Ichinose, M.: J. Reine Angewdt. Math. 244, 119-153 (1970)

18. Kako, T., Yajima, K.: Sci. Papers Coll. Gen. Educ. Univ. Tokyo 26, 73-89 (1976)

19. Kato, T.: Math. Ann. 162, 258-279 (1966)

20. Kato, T. : J. Fac. Sci. Univ. Tokyo, Sect. IA 24, 503-513 (1977)

21. Kuroda, S.T.: Analyse Math. 20, 57-117 (1967)

22. Kuroda, S.T. : J. Math. Soc. Jpn. 25, 75-104; 222-234 (1973)

23. Kuroda, S.T.: An introduction to scattering theory. Aarhus Univ., Lecture Notes Series 51 (1979)

24. Lavine, R.: Indiana Univ. Math. J. 21, 643-656 (1972)

25. Nuttall, J., Singh, S.R.: Univ. W. Ontario, Preprint (1979)

26. Reed, M., Simon, B.: Methods of modern mathematical physics IV. London, New York: Academic Press (1978)

27. Sigal, I.M. : Mem. Am. Math. Soc. 16, 209 (1978)

28. Sigal, I.M. : Bull. Am. Math. Soc. 84, 1, 152-154 (1978)

29. Simon, B.: Commun. Math. Phys. 27, 1-12 (1972)

30. Simon, B.: Commun. Math. Phys. 55, 259-274 (1977); 58, 205-210 (1978)

31. Steinberg, S.: Arch. Rat. Mech. Anal. 31, 372-379 (1968)

32. Van Winter, C.: J. Math. Anal. Appl. 47, 633-670; 48, 368-399 (1974)

33. Van Winter, C.: J. Math. Anal. Appl. 49, 88-123 (1975)

34. Van Winter, C.: Notices Am. Math. Soc. 25, A-320 (1978)

35. Yajima, K.: J. Fac. Sci. Univ. Tokyo, Sect. IA 25, 109-132 (1978)

Communicated by J. Ginibre

Received November 13, 1979 